Florida International University

FIU Digital Commons

FIU Electronic Theses and Dissertations

University Graduate School

$12-4-2019$

\title{
Associations Between Adolescent Cannabis Use Trajectories and Anxiety
}

Jacqueline C. Duperrouzel

jduperro@fiu.edu

Follow this and additional works at: https://digitalcommons.fiu.edu/etd

Part of the Clinical Psychology Commons

\section{Recommended Citation}

Duperrouzel, Jacqueline C., "Associations Between Adolescent Cannabis Use Trajectories and Anxiety" (2019). FIU Electronic Theses and Dissertations. 4535.

https://digitalcommons.fiu.edu/etd/4535

This work is brought to you for free and open access by the University Graduate School at FIU Digital Commons. It has been accepted for inclusion in FIU Electronic Theses and Dissertations by an authorized administrator of FIU Digital Commons. For more information, please contact dcc@fiu.edu. 


\section{FLORIDA INTERNATIONAL UNIVERSITY}

Miami, Florida

\section{ASSOCIATIONS BETWEEN ADOLESCENT CANNABIS USE TRAJECTORIES AND ANXIETY}

A dissertation submitted in partial fulfillment of the requirements for the degree of DOCTOR OF PHILOSOPHY

in PSYCHOLOGY

by

Jacqueline Christine Duperrouzel 
To: $\quad$ Dean Michael R. Heithaus

College of Arts, Sciences and Education

This dissertation, written by Jacqueline Christine Duperrouzel, and entitled Associations Between Adolescent Cannabis Use Trajectories and Anxiety, having been approved in respect to style and intellectual content, is referred to you for judgement.

We have read this dissertation and recommend that it be approved.

Stefany Coxe

Jonathan Comer

Wasim Maziak

Elisa Trucco

Raul Gonzalez, Major Professor

Date of Defense: December 4, 2019

The dissertation of Jacqueline Christine Duperrouzel is approved.

Dean Michael R. Heithaus

College of Arts, Sciences and Education

Andrés G. Gil

Vice President for Research and Economic Development

And Dean of the University Graduate School

Florida International University, 2020 
(C) Copyright 2020 by Jacqueline Christine Duperrouzel

All rights reserved. 


\section{DEDICATION}

I dedicate this thesis to my parents Christine and Phillippe Duperrouzel, without their love, patience, and support this work would not have been possible. I additionally want to thank my friends and extended family, the Gunia's, Nasralla's, Lascelle's, Duperrouzel's, Azan's, Mahfood's, Feanny's, \& Buddle's for their unconditional love and support. Lastly, thank you Joseph for always encouraging me to be the best, I love you. 


\section{ACKNOWLEDGMENTS}

I wish to thank the members of my committee for their support, patience, and good humor. Their gentle but firm direction has been most appreciated. To my mentor, Raul Gonzalez, thank you for guiding me these past five years on my journey of becoming a neuropsychologist. To my best friend in the SUHN lab, Ileana Pacheco-Colón, you have

made this journey so much more brighter and fun. Lastly, thank you Samuel Hawes, for your patience and guidance through my statistical training these past few years, it has been a pleasure learning from you. 


\author{
ABSTRACT OF THE DISSERTATION \\ ASSOCIATIONS BETWEEN ADOLESCENT CANNABIS USE \\ TRAJECTORIES AND ANXIETY \\ by \\ Jacqueline Christine Duperrouzel \\ Florida International University, 2020 \\ Miami, Florida

\section{Professor Raul Gonzalez, Major Professor}

Understanding the effects of cannabis use is critical for reducing adverse behavioral, social, and academic outcomes, particularly among adolescent users who are most at risk for cannabis related problems. Although support from both the animal and human literatures suggests the relationship between cannabis and anxiety may be associated with frequency of use, much is still unknown. Thus, examining relationships between the most common mental health issue in adolescence and one of the most commonly used drugs is of great public health significance and impact. Prior longitudinal studies assessing effects of cannabis use on anxiety have not evaluated different patterns of use, limiting our ability to identify who may be most at risk for poorer anxiety outcomes. Assessing the impact of cannabis trajectories on prospective anxiety will allow us to answer the question of specificity, and the role different cannabis patterns have on changes in anxiety symptoms and disorders. The current study leveraged data from a large NIDA-funded study (R01 DA031176) to examine associations between adolescent cannabis use patterns and changes in anxiety symptomology, disorder development, and the interactive effects on decision-making, with data collected from 401 
teens across two years. We employed advanced latent growth curve modeling techniques within an accelerated cohort framework, to allow for additional examination of the effects patterns of cannabis use have on anxiety during mid to late adolescence. Three cannabis use trajectories emerged, including a minimal, escalating, and chronic use trajectory. Overall, findings suggest cannabis use during adolescence is associated with changes in anxiety over time. Specifically, past week anxiety symptoms increased in escalating and chronic users relative to minimal users at the two-year follow up. Surprisingly, less cannabis use was associated with greater likelihood of anxiety disorder development and several hypotheses are provided as to why this may occur. Further, cannabis trajectory did not influence risky decision-making independently, but rather interacted with anxiety to influence risk-taking among minimal users. Overall, the results of the present study were the first to identify how trajectories of cannabis use influence prospective anxiety in a sample of adolescents across a two-year time span. Our findings contribute to prevention and intervention efforts by identifying adolescent users who are most at risk for developing anxiety symptoms and making poor decisions. Future interventions that target reducing anxiety and cannabis cessation concurrently will further improve cognitive functioning among heavy users. 
I. INTRODUCTION TO THE RESEARCH

II. LITERATURE REVIEW OF ADOLESCENCE, CANNABIS,

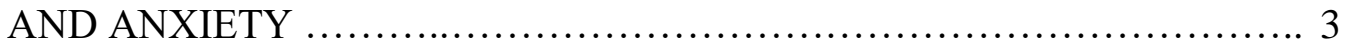

Importance of Examining Adolescent Cannabis Use ....................... 3

Significance and Effects of Anxiety in Adolescence ................... 5

Associations between Cannabis Use, Anxiety, and the Brain ............ 8

Summary \& Proposed Study ....................................... 12

III. METHODOLOGY .............................................. 13

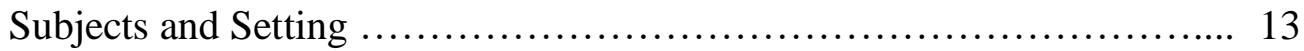

Measures ........................................................ 15

Statistical Analyses ............................................... 19

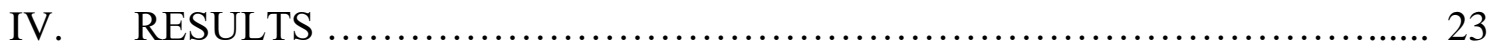

Descriptive Statistics .......................................... 23

Aim 1 Result Part A: LCGA of Cannabis Use Trajectories................ 24

Aim 1 Results Part B: Cannabis Trajectories and Change in Anxiety........ 26

Aim 2 Results: Odds of Developing and Anxiety Disorder at T3 or T5.... 27

Aim 3 Results: Anxiety as Moderator of the Relationship between

Cannabis Use Trajectories and Decision-Making .................... 31

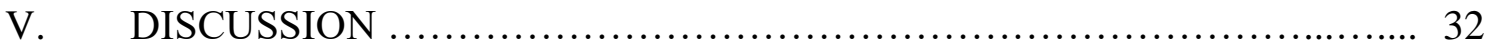

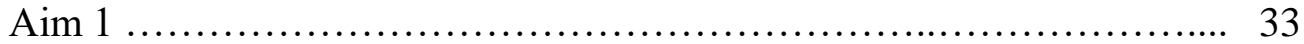

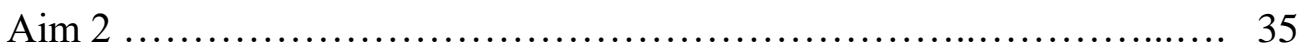

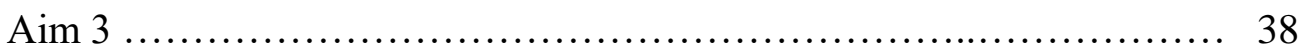

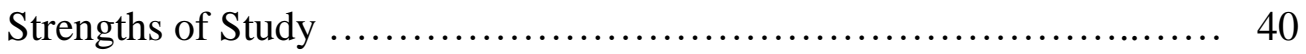

Limitations ....................................................... 43

Conclusions and Future Directions ............................. 46

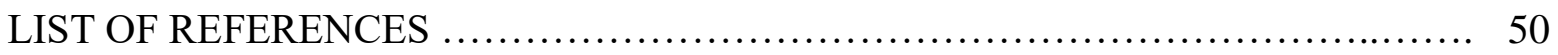

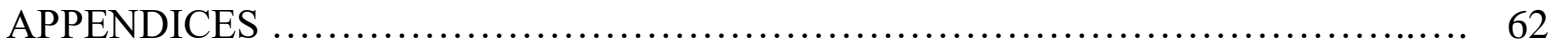

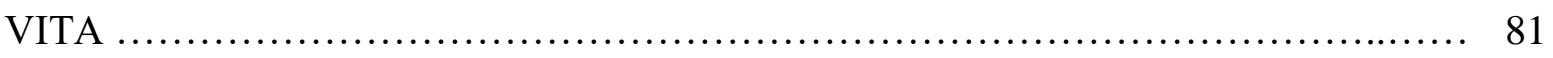




\section{LIST OF TABLES}

TABLE

PAGE

1. Descriptive Statistics for Study Sample at Baseline $\ldots \ldots \ldots \ldots \ldots \ldots \ldots \ldots \ldots \ldots \ldots$

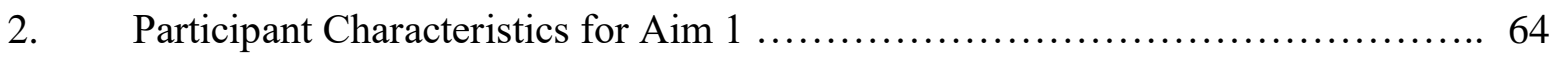

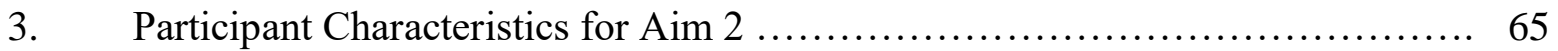

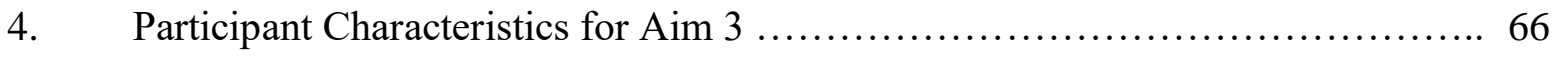

5. Fit Information for Latent Class Growth Analyses of Cannabis Trajectories ..... 67

6. Results of cannabis use trajectory membership predicting changes in anxiety

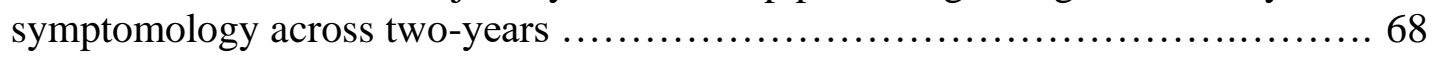

7. Results of cannabis use trajectory membership predicting panic disorder and general anxiety disorder membership at 1-year and two-year follow-up . ........ 69

8. Model parameter estimates of the interactive effects of anxiety and cannabis use trajectory membership with decision-making at two-year follow-up ............. 70 


\section{LIST OF FIGURES}

FIGURE

PAGE

1. Proposed Biopsychosocial Model of Anxiety and Cannabis Use 71

2. Reinforcement Model of Anxiety through Self-Medicating with Cannabis Use ... 72

3. Assessment Timeline from Baseline to T5 .............................. 73

4. Trajectories of Adolescent Cannabis Use at Baseline - Three Classes ........... 74

5. Trajectories of Adolescent Cannabis Use at Baseline - Four Classes ........... 75

6. Changes in DASS-21 Anxiety Symptoms by Cannabis Use Trajectory From T1-T5 ........................................................ 76

7. Changes in PD Symptoms Anxiety by Cannabis Use Trajectory from T1-T5 _.... 77

8. Changes in GAD Symptoms by Cannabis Use Trajectory from T1-T5 $\ldots \ldots \ldots \ldots . .78$

9. Mean Unstandardized Anxiety Residuals by Cannabis Use Trajectory ........... 79

10. Main Effects of Anxiety and Cannabis Use Trajectory Interaction on Decision-Making 80 


\section{ABBREVIATIONS AND ACRONYMS}

\begin{tabular}{|c|c|}
\hline BOLD & Blood Oxygen Level Dependent \\
\hline CDISC & Computerized Diagnostic Interview Schedule for Children \\
\hline CNS & Central Nervous System \\
\hline $\mathrm{CT}$ & Cups Task \\
\hline CUD & Cannabis Use Disorder \\
\hline DASS- 21 & Depression, Anxiety, Stress Scale, short version \\
\hline DUHQ & Drug Use History Questionnaire \\
\hline fMRI & Functional Magnetic Resonance Imaging \\
\hline GAD & General Anxiety Disorder \\
\hline GDT & Game of Dice Task \\
\hline IGT & Iowa Gambling Task \\
\hline LCGA & Latent Class Growth Analysis \\
\hline MPA & Maximum Probability Assignment \\
\hline MRI & Magnetic Resonance Imaging \\
\hline MTFS & Monitoring the Future Survey \\
\hline NIDA & National Institute of Drug Abuse \\
\hline $\mathrm{NIH}$ & National Institute of Health \\
\hline NP & Neuropsychological \\
\hline PD & Panic Disorder \\
\hline $\mathrm{THC}$ & Delta-9-tetrahydrocannabinol \\
\hline
\end{tabular}




\section{CHAPTER I.}

\section{INTRODUCTION TO THE RESEARCH}

The program of research that was created for this dissertation examines the effects of unique patterns of adolescent cannabis use with changes in recent levels of anxiety (i.e., past week), panic disorder, and general anxiety disorder symptoms and disorder development. Due to neuromaturational changes occurring in the adolescent developmental period, it is hypothesized that increasing and high levels of cannabis use during this time of development affect emotional and cognitive functioning. The current study first highlights the extant literature on adolescent cannabis use, anxiety, and the established associations between these two topics. This is followed by preliminary findings published in the journal Addictive Behaviors to provide rationale for further exploration of the associations between cannabis use and anxiety. Next, additional exploration of associations between cannabis use and anxiety are conducted and guided by my three aims and hypotheses. Aim 1: Examine trajectories of cannabis use among adolescents and their associations with changes in anxiety symptoms. (1a) Unique trajectories of adolescent cannabis use trajectories will emerge. Next,trajectories will be associated with unique increases in anxiety symptoms, such that the minimal-stable group will be show the lowest increase in anxiety relative to the other trajectories, whereas escalating users will show the highest increase, and high-stable users will be in-between. Aim 2: Examine associations between cannabis use trajectories and the development of an anxiety disorder. (2a) Different use trajectories will be associated with different odds of developing an anxiety disorder, with users in the escalating trajectory having the greatest risk of anxiety disorder development. Aim 3: Explore whether anxiety moderates 
the relationship between cannabis use trajectories and DM. (3a): Anxiety will moderate associations between cannabis use and decision making, such that for teens with higher levels of anxiety, heavier patterns of CU (i.e., escalating and chronic trajectories) will be associated with riskier DM. With lower levels of anxiety, the association between cannabis use trajectories and DM will be diminished or reversed, such that heavier patterns of CU (i.e., escalating and chronic trajectories) will be associated with better (i.e., less risky) DM. Lastly, results of these analyses are provided and discussed in the context of intervention and prevention efforts. 


\section{CHAPTER II.}

\section{Literature Review of Adolescence, Cannabis, and Anxiety}

\section{Importance of Examining Adolescent Cannabis Use}

Cannabis is among the most widely abused substance by adolescents after alcohol in the United States, (Johnston et al., 2018), yet many youths do not perceive it as a harmful substance (Brook, Lee, Brown, Finch, \& Brooke, 2011; Hayatbakhsh et al., 2007). According to the 2016 Monitoring the Future Study (MTFS), the perception of risk associated with smoking cannabis has continued to decline within the past decade, with roughly $31 \%$ of high-school seniors reporting regular use as harmful, compared to $58 \%$ in 2000 . Despite low risk perception, consequences of adolescent cannabis use include difficulties in academic, social, occupational, and mental health domains (Fergusson \& Boden, 2008; Lynskey \& Hall, 2000; Volkow, Baler, Compton, \& Weiss, 2014;). As of March 2019, 33 states and the District of Columbia have legalized cannabis for medicinal purposes (NCSL, 2019). Among states with current medical and recreational cannabis laws, rates of use among youth remain higher when compared to states without laws not allowing cannabis use (Wadsworth \& Hammond, 2018). Although prior research on the neuroprotective properties of cannabinoids (i.e., antiinflammatory, anti-excitotoxic, antioxidant effects) (Baker, Price, Giovannoni, \& Thompson, 2003; Hampson et al., 2000; Van der Stelt et al., 2001) have informed these policies, the harmful effects on brain function and associated mental health symptoms of cannabis on the developing brain are still largely unknown.

It is hypothesized that adolescents may be particularly sensitive to adverse effects of cannabis because of changes in central nervous system development including 
myelination, synaptic pruning, and structure maturation (Batalla et al., 2013; Lubman, Cheetham, \& Yücel, 2015; Squeglia, Jacobus, \& Tapert, 2009). In addition to adolescence being a critical period of neuromaturation, changes in physical, emotional, and social functioning are occurring simultaneously (Steinberg, 2005). The rapid changes occurring across social and biological domains place adolescents at heightened risk for initiating risky behaviors (Squeglia \& Tapert, 2009). There are several relevant and competing theories as to why adolescents initiate and abuse substances during this developmental period. Proposed by Bronfenbrenner (1979, 2005), the bioecological model of development states that as children develop, they interact with four systems: the microsystem (i.e., individual level factors), mesosystem (i.e., relationship level factors), exosystem (i.e., community level factors), and macrosystem (i.e., society level factors). When an adolescent's personal factors (e.g., rebelliousness) interact with the other three system factors (e.g., peer pressure (i.e., mesosystem), exposure and access to substances (i.e., exosystem) and culture/perceived risk (i.e., macrosystem)), it is hypothesized that substance use occurs (Dodge et al., 2009). Another relevant theory for adolescent substance use is the dual systems model of cognitive development proposed by Steinberg and colleagues (2013), which suggests increased risk-taking occurs during this time because of an imbalance in neural development of certain brain structures, which leads to heightened reward sensitivity and immature impulse control (Strang, Chein, \& Steinberg, 2013). Lastly, a self-medicating hypothesis indicates that substance use may be a maladaptive coping mechanism for stress and negative emotional states (Khantzian, 1997). Although these theories provide insight into possible factors that influence early substance use, determining causal mechanisms are still limited. Indeed, emerging studies 
reveal that adolescence is a period of heightened risk taking and the most common period for initiation of cannabis use (Chen \& Kandel, 1995), yet longitudinal examination on prospective effects is limited. While adolescent patterns of cannabis use are varied, typical trajectories often include an escalating trajectory in which an individual increases use over time; a high stable user, in which an individual maintains moderate to high use over time; and a low stable user, in which an individual maintains low use over time (Schulenberg et al., 2005). Understanding the effects of how distinct patterns of use may impact different behavioral and neural processes could have the potential to inform prevention efforts. Accordingly, focused efforts are needed to investigate the effects of cannabis use on cognitive, social, and emotional domains to help inform prevention and intervention practices for the well-being of our youth. Thus, given previously established associations between cannabis use and mental health problems (Crippa et al., 2009; Kedzior \& Laeber, 2014; Patton et al., 2002), research is needed to elucidate trajectories that may lead to adverse psychological and cognitive outcomes.

\section{Significance and Effects of Anxiety in Adolescence}

Research indicates that anxiety disorders are the most commonly diagnosed adolescent psychiatric conditions (Conway, Swendsen, Husky, Je, \& Merikangas, 2016). It is estimated that approximately $31.9 \%$ of adolescents suffer from at least one of the five major types of anxiety disorders, which include panic, social anxiety, separation anxiety, generalized anxiety, and specific phobia disorders (Conway et al., 2016). Symptoms of anxiety disorders include worry and physiological arousal, such as restlessness, insomnia, and muscle tension (Beesdo, Knappe, \& Pine, 2009). As a result, anxiety disorders account for decreased productivity, increased morbidity and mortality 
rates, and increased alcohol and drug abuse (King, Iacono, \& McGue, 2004).

Furthermore, past findings suggest that the presence of an anxiety disorder in adolescence increases the risk of secondary psychopathology development, potentially contributing to educational underachievement and functional impairments (Woodward \& Fergusson, 2001). Currently, as detailed by a large body of literature, the most common anxieties in adolescence are specific phobia and social anxiety disorder (Beesdo et al., 2009; Merikangas et al., 2010). However, there is a growing literature on the development and prospective effects of generalized anxiety disorder (GAD) and panic disorder (PD) in adolescence. According to the Diagnostic and Statistical Manual of Mental disorder, Fifth Edition (DSM-5), GAD is described as a condition characterized by excessive anxiety and worries, on most days, for at least six months and causing significant distress in social, occupational, and other areas of functioning (American Psychiatric Association, 2013). Among adolescents with GAD, alterations in brain connectivity have been observed in regions of the brain associated with emotional processing (i.e., amygdala, insula, temporal gyrus, cerebellum, and putamen) when compared to healthy non-anxious adolescents (Lie et al., 2015). These abnormalities in blood-oxygenated level dependent (BOLD) signal have been identified during resting state fMRI data acquisition (Lie et al., 2015), as well as during cognitive task performance (Monk et al., 2008) as demonstrated by increased BOLD signal within these brain regions. Additionally, findings suggest that adolescents with severe symptoms of GAD (e.g., high scores modeled by the Pediatric Anxiety Rating Scale) additionally showed decreased BOLD activation in the prefrontal cortex when compared to adolescents with mild symptoms (Monk et al., 2008). Similarly, patterns of decreased BOLD levels have been observed in the medial 
prefrontal cortex in patients with PD when compared to healthy matched controls (Zhao et al., 2007). Described by the American Psychiatric Association (2013), PD is characterized by recurrent panic attacks, with one or more attacks followed by at least a month of fear related to panic attacks. The implications of GAD and PD symptomology on BOLD within the prefrontal cortex may be translational and observed in decisionmaking (DM) and higher order executive functioning, thus impacting an adolescent's overall cognitive abilities.

Comparable to the documented cannabis use trajectories, developmental patterns of anxiety have been studied. Prior research indicates that anxiety follows a natural waxing and waning profile across adolescence (Kessler, Ruscio, Shear, Wittchen, 2009). Yet, disparities between anxiety disorder growth and reduction rates have revealed differences between PD and GAD, such that PD symptoms decrease in early to middle adolescence compared to increases in GAD symptomology (Hale III, Raaijmakers, Muris, \& Meeus, 2008) during the same time period. Furthermore, although anxiety disorders are common among teens, many individuals do not seek treatment (Coles \& Coleman, 2010), despite established effective therapeutic strategies (Walkup et al., 2008). Rather, individuals with earlier onset (Douglas et al., 2010) and untreated anxiety are at greater risk for mood difficulties and substance use (Brown, Campbell, Lehman, Grisham, \& Mancill, 2001). Such findings highlight the need to better understand and address the unique mechanisms that contribute to the development of distinct anxiety disorders among youths, as well as, what propagates maladaptive coping mechanisms, such as substance abuse, among youth. 


\section{Associations between Cannabis Use, Anxiety, and the Brain}

A biopsychosocial conceptualization of how anxiety and cannabis use may be related include evaluating physiological arousal, cognitive thoughts, and feelings within the context of an individual's life (See Figure 1). Although it is proposed that biological, social, and psychological factors interact to influence the relationship between anxiety and cannabis, we suggest that the association is influenced by a negative reinforcement model of anxiety (See Figure 2). Our proposed reinforcement model for using cannabis may be attributed to an individual's attempt to regulate negative emotional and physiological states in the short term (Buckner, Heimberg, Ecker, \& Vinci, 2013), rather than regulating stress and worries via evidence-based methods (e.g., cognitive restructuring and relaxation techniques). Additionally, utilizing cannabis as a coping mechanism for anxiety may be influenced by social pressures (Buckner, Bonne-Miller, Zvolensky, \& Schmidt, 2007), genetic underpinnings (Merikangas \& Avenevoli, 2000), and psychological traits (Kotov, Gamez, Schmidt, \& Watson, 2010).

Another viable factor for the maintenance of comorbid cannabis use and anxiety within the biopsychosocial framework is comparable neural activations within emotional processing regions of the brain (Lantela, Kamal, \& Kamal, 2018). Cannabis exerts its effects on the central nervous system (CNS) through the binding of its primary psychoactive constituent delta-9-tetrahydrocannabinol (THC) to cannabinoid 1 (CB1) receptors distributed throughout the brain (Iversen, 2003). There is significant overlap between brain structures with large densities of CB1 receptors and those implicated with processes of anxiety, thus providing a viable mechanism through which cannabis use may impact anxiety symptomology. Specifically, the amygdala and prefrontal cortex-brain 
regions recruited in anxiety and executive functioning processes which include planning and DM (Blakemore \& Robbins, 2012; Ochsner \& Gross, 2005)—are known to have a high density of CB1 receptors (Iversen, 2003; Ochsner \& Gross, 2005; Rey, Purrio, Viveros, \& Lutz, 2012). Although cannabis use may reduce subjective anxiety acutely, the picture is more complex. Cannabis is known to have a dose-biphasic effect with high levels of THC resulting in symptoms of acute anxiety and low levels resulting in an anxyioltic effect (Rey et al., 2012). Research conducted in rodents shows that chronic exposure to cannabis during adolescence can influence neural microstructure by reducing CB1 receptor levels in cortical brain regions (Hirvonen et al., 2012; O'shea, Singh, McGregor, \& Mallet, 2004; Rubino et al., 2007). The downregulation of receptors is hypothesized to exacerbate anxiety, as normal endocannabinoid signaling is disrupted (Katona et al., 2001; Martin, Ledent, Parmentier, Maldonado, \& Valverde, 2002). Discovered in 1990s, the endocannabinoid system can be found in the central and peripheral nervous systems and aids in several physiological and cognitive processes including motivation, control, and reward, (Maldonado, Valverde, \& Berrendero, 2006). Mirrored in human literature, an adult study utilizing positron emission tomography (PET) revealed a $20 \%$ reduction of CB1 receptors across the brain in heavy users when compared to those with limited levels of cannabis exposure across their life (Hirvonen et al., 2012). The anatomical disruption of CB1 receptor density, in conjunction with exogenous cannabinoid consumption could potentially influence neurotransmitter, chemical, and hormonal signaling, thereby contributing to the development of psychological and mood disorders (Ashton \& Moore, 2011). These effects observed after long-term use suggest that regular cannabis use may impact neurobiological processes 
relevant to anxiety (Braida, Limonta, Malabarba, Zani, \& Sala, 2007; Muntoni et al., 2006; Viveros, Marco, \& File, 2005) and influence neuropsychological functioning including DM (Crean, Crane, \& Mason, 2011). Although downregulation of CB1 receptors with chronic use may be reversible upon cessation of use (Hirvonen et al., 2012), it is not clear whether that is the case when use occurs during adolescence. Moreover, disruptions in receptor densities during a period marked by heightened neural development may make adolescents more susceptible to long-lasting receptor disruptions (Lubman et al., 2015; Schneider, 2008) and more severe consequences of cannabis use.

Recently, there has been accumulating evidence suggesting heavy cannabis use during adolescence is associated with social anxiety disorder (SAD), the most commonly studied anxiety disorder among adolescent cannabis-using samples (Buckner, Schmidt, Bobadilla, \& Taylor, 2006; Buckner et al., 2007; Buckner et al., 2008; Buckner, Heimberg, Mathews, \& Silgado, 2012; Buckner et al., 2012). Despite insights on the association between cannabis use and $\mathrm{SAD}$, little is known on how various patterns of use affect other anxiety symptomology over time, specifically with regards to PD and GAD. Historically, cannabis use has been associated with symptoms of panic (Tunving, 1985). These findings have influenced a relatively small line of research that explores the dose-dependent effects of cannabis on physiological symptoms of anxiety. Findings from these studies have provided support for the association of heavier use with acute panic attacks (Crippa et al., 2009; Szuster, Pontius, \& Campos, 1988) and future development of PD (Zvolensky, Lewinsohn, Bernstein, Schmidt, \& Buckner, 2008). Accordingly, research examining GAD with cannabis use is scarce. Of interest, one longitudinal study found that any cannabis (i.e., > 1x time) use is associated with increased PD and GAD 
development compared to individuals who do not use cannabis (Van Laar, Van Dorsselaer, Monshouwer, \& De Graaf, 2007).

Additionally, chronic cannabis use in adolescence has been associated with cognitive impairment (Meier et al., 2012), but examination of the interactive effects of adolescent cannabis use and anxiety on domains of cognitive functioning has not occurred. Furthermore, longitudinal studies evaluating associations among cannabis use trajectories and changes in anxiety symptoms and anxiety disorder development are scarce. More information regarding the factors that contribute to associations between anxiety and cannabis use warrants additional exploration.

Preliminary data from our lab published in the journal Addictive Behaviors, identified that early levels of cannabis use influence rates of change in self-reported past week anxiety symptoms in a sample of 250 adolescents across a one-year period (Duperrouzel et al., 2018). Data on participants' past month cannabis frequency and past week's subjective anxiety symptoms informed this relationship. Our prior work suggests greater levels of cannabis use are associated with a slower decrease in anxiety symptoms over time (relative to the sample average) in adolescents, even after accounting for concurrent depression, alcohol, and nicotine use $(\beta=15, p<.05)$. In addition, our results highlight differences between varying levels of cannabis use and anxiety symptoms, lending support to theoretical models that support the theory that early onset substance use impacts mental health (Wittchen et al., 2007). However, whether the maintenance of this association persists across larger samples and time-frames, as well as whether this association generalizes to other anxiety disorder symptoms remain unknown. Given these 
gaps in our understanding of adolescent cannabis use and anxiety, it is timely to examine these associations.

\section{Summary and Proposed Study}

Although relationships between psychopathology and cannabis use is being ardently studied, especially with regards to psychosis and schizophrenia (Brook et al., 2011; Hall \& Degenhardt, 2000; Hayatbakhsh et al., 2007), associations with nonpsychotic disorders have received far less attention. An area receiving increasing attention is the association between cannabis use and anxiety. Understanding the effects of cannabis use is critical for reducing adverse behavioral, social, and academic outcomes, particularly among adolescent users who are most at risk for cannabis related problems. Although support from both the animal and human literature suggests the relationship between cannabis and anxiety may be influenced by levels of use, much is still unknown. Thus, examining relationships between the most common mental health issue in adolescence and one of the most commonly used drugs is of great public health significance and impact. Prior longitudinal studies assessing effects of cannabis use on anxiety have not extensively evaluated different patterns of use, limiting our ability to identify who may be most at risk for poorer anxiety outcomes. The need to assess cannabis trajectories with changes in anxiety over time will allow us to answer the question of specificity (i.e., who is more adversely affected) and the role that different cannabis patterns have on prospective anxiety symptom and disorder development. Findings from this research are expected to advance our understanding of the association between cannabis use and anxiety. Thus, the aims of this dissertation are: Aim 1:

Examine trajectories of cannabis use among adolescents and their associations with 
changes in anxiety symptoms. (1a) Previously identified trajectories will be associated with unique increases in anxiety symptoms, such that the minimal-stable group will be show the lowest increase in anxiety relative to the other trajectories, whereas escalating users will show the highest increase, and high-stable users will be in-between. Aim 2. Examine associations between cannabis use trajectories and the development of an anxiety disorder. (2) Different use trajectories will be associated with different odds of developing an anxiety disorder, with users in the escalating trajectory having the greatest risk of anxiety disorder development. Aim 3: Explore whether anxiety moderates the relationship between cannabis use trajectories and DM. (3): Anxiety will moderate associations between cannabis use and decision making, such that for teens with higher levels of anxiety, heavier patterns of CU (i.e., escalating and chronic trajectories) will be associated with riskier DM. With lower levels of anxiety, the association between cannabis use trajectories and DM will be diminished or reversed, such that heavier patterns of CU (i.e., escalating and chronic trajectories) will be associated with better (i.e., less risky) DM..

\section{CHAPTER III.}

\section{METHODOLOGY}

\section{Subjects and Setting}

My study leveraged data from a NIDA funded project (R01DA031176 PI: Gonzalez) examining the neurocognitive effects of adolescent cannabis use. Participants are 401 adolescents at risk for cannabis use escalation from the greater Miami area. They were recruited via in-person recruitment efforts at Miami-Dade County public middle and

high schools, flyers distributed throughout South Florida, and word-of-mouth. Adolescent 
eligibility was ascertained via phone screen, and parental verbal and written consent were obtained prior to assessment scheduling. Participants in the study were all between the ages of 14-17 at the time of their baseline evaluation and were able to read and write in English. By design, most had previous exposure to either alcohol, cigarettes, or other drugs (even if only minimal), Participation exclusionary criteria included self-reported 1) developmental disorders (e.g., delays in motor and language abilities), 2) neurological conditions (e.g., stroke, brain aneurism, seizures, epilepsy), 3) birth complications (e.g., born <37 week-gestation), 4) history of self-reported diagnosis of mood disorder or thought disorder at screening, 5) traumatic brain injury or loss of consciousness $>10$ minutes, 6) history of significant alcohol use or substance use suggestive of an alcohol use disorder (AUD) or cannabis use disorder (CUD), 7) use of other drugs (besides alcohol, cannabis, and nicotine) more than 10 times, 8) use of any other drugs in the two weeks prior to assessment, and 9) use of any other drug to an extent greater than their cannabis use. Participants completed a detailed baseline assessment by trained examiners that included measures of substance use, executive functioning, risky behaviors, and mood. Five measurement waves of data were conducted bi-annually for two years. The baseline and 1-year follow-up assessments (T3 and T5) queried all aforementioned areas, as well as, oral toxicology and breath tests for assessment of trace levels of recent drug use. All one-year follow up assessments were conducted at a laboratory during a visit lasting approximately 4 hours. The 6-month follow-up assessments (T2 and T4) were conducted through a telephone call lasting approximately one hour and focused on past 6month substance use and mood symptoms. Data collected for aims 1 and 2 utilized measures administered at all five assessment waves, whereas the aim 3 DM data 
examined all in-person assessments (i.e., 3 waves of data), see Figure 3. Participant characteristics for the sample are presented in Tables 1-4. The Institutional Review Board of Florida International University (FIU) approved all study procedures and protocols (IRB-13-0065-AM37).

\section{Measures}

Self-report measures, structured and semi-structured interviews, and computer based decision-making tasks were utilized to assess substance use, mood, and DM. All data was uploaded to a secure encrypted online platform (REDCap).

Demographic Information. Information regarding participant's age, race, ethnicity, gender, years of education, parental education, birth, medical, and substance use history were collected via the clinician-administered Personal History Questionnaire (PHQ) at each assessment wave.

Toxicology Testing. All participants underwent breath alcohol and carbon monoxide (CO) testing at baseline, T3, and T5 (BACtrack S80; piCO+ Smokerlyzer coVita). The alcohol breath test assessed for alcohol concentrations between $0.00-.500 \%$ within 3 seconds. Whereas, the CO testing detected for levels of $0-150$ ppm CO in <30 seconds. Additionally, participants underwent oral fluid toxicology testing for recent drug use of THC, cocaine, opiates, and amphetamines using the Intercept oral fluid drug test at all in-person assessments (OraSure Technologies, Inc.: Bethlehem, PA; Langel et al., 2008).

Substance Use. Detailed substance use history was obtained using the Drug Use History Questionnaire (DUHQ) (Duperrouzel et al., 2019; Rippeth et al., 2004), a semistructured interview assessing self-reported frequency and quantity of substance use 
across 15 different classes of substances during a participant's lifetime, past six months, and past 30 days. In addition to cannabis use, data were obtained on alcohol, nicotine, cocaine, amphetamines, methamphetamine, ecstasy, heroin, opiates, benzodiazepines, barbiturates, club drugs, hallucinogens, PCP, and inhalants. Cannabis use frequency (i.e., number of days used) from the past six months will be our primary measure for generating cannabis use trajectories for our analyses.

Anxiety Symptoms Measures. Anxiety symptoms were assessed through a selfreport questionnaire and a semi-structured interview.

Past week symptoms of anxiety were assessed using the short version of the Depression, Anxiety, Stress Scale (DASS-21; Henry \& Crawford, 2005), a 21-item selfreport questionnaire used to assess symptoms of depression, anxiety, and stress during the past week. Items are rated on a 4-point scale, ranging from 0 ("Did not apply to me at all") to 3 ("Applied to me very much, or most of the time"). Evidence of reliability and validity of the DASS-21 ( $\alpha=0.81 ; 95 \%$ CI: $0.79-0.84)$ among adolescents has been well established (Szabó, 2010). Seven statements assessing anxiety included awareness of dryness of mouth, breathing problems, action of heart, trembling, feeling worried, feeling close to panic, and feeling scared. The current study used this measure's 7-item anxiety subscale standardized z-scores as an outcome measure of anxiety. Stress and depression subscale z-scores were controlled for in follow-up analyses and used as covariates for Aims 1-3.

The Computerized Diagnostic Interview Schedule for Children (C-DISC), a structured reliable interview for children and adolescents (Shaffer, Fisher, Lucas, Dulcan, \& Schwan-Stone, 2000), was used to identify the following disorders: panic disorder, 
general anxiety disorder, obsessive compulsive disorder, major depression disorder, mania/hypomania, attention deficit/hyperactivity disorder, oppositional defiant disorder, and conduct disorder at all in-person assessment waves (i.e., baseline, 1-year follow-up (T3), and 2-year follow-up (T5)). The C-DISC also provides number of symptoms endorsed with each disorder, impairment level, and sub-clinical intermediate diagnoses. PD and GAD symptoms and disorder diagnosis were used as anxiety outcome variables for Aims 1 and 2.

\section{Decision-Making.}

At all in-person visits (baseline, T3, and T5) decision-making (DM) was examined with three computerized tasks assessing DM under various conditions of risk

and reward. Structural equation modeling was employed to generate a latent construct of DM performance at T5 with data from each of the DM tasks (Pacheco-Colón, Hawes, Duperrouzel, Lopez-Quintero \& Gonzalez, 2019).

The Iowa Gambling Task (IGT) was used to assess DM under ambiguous risk (Bechara, Damasio, Damasio, \& Anderson, 1994). Participants completed 100 trials in which they had to select from four card decks, which included two "good" (i.e., Decks C and D) and two "bad" (i.e., Decks A and B) decks, in an attempt to yield a positive net total of monetary earnings. Participants were told that when they select a card, they will win money, sometimes lose money, and that some decks are better than others. To obtain a positive net total at game completion, a participant would had to have made more selections from "good" rather than "bad" decks. The Net Score was derived from the selection of bad decks subtracted from the selection of good decks and was used as an indicator for the DM latent variable. As recommended in the IGT Professional Manual, 
we reverse scored the Net Score to aid with interpretation of findings (Bechara, 2007) in our analyses. Prior research utilizing the IGT with individuals that use cannabis found that poor DM moderated relationships, between cannabis use and risky behaviors; such that at high levels of cannabis use better DM was associated with less risky behaviors, whereas poorer DM was associated with increased risk-taking (Ross, Coxe, Schuster, Rojas \& Gonzalez, 2015).

Additionally, the Game of Dice Task (GDT) was used to assess DM under explicit risk probabilities (Brand et al., 2005). Participants made selections for simulated monetary gain by evaluating the probability of winning or losing across 18 trials. During the GDT, combinations of one to four dice are presented and participants were instructed to pick one of the combinations presented on each trial. Probabilities of earning a specific outcome are associated with the number of dice selected, with high risk choices (i.e., 1 or 2 dice options). associated with higher gains but greater probabilities for large losses. Alternatively, low risk choices are associated with higher probabilities of winning smaller gains (i.e., 3 or 4 dice options), but with the risk of smaller possible losses. Total number of risky trials was used as an indicator for the DM latent variable.

Lastly, the Cups Tasks (CT) was used to assess DM under conditions of specified risk separately for conditions of gain and loss (Levin \& Hart, 2003; Levin, Hart, Weller, \& Harshman, 2007). Participants were instructed to pick a cup from one of two sides that would either yield a definite reward/smaller loss, or from the opposite side that provided a chance for a greater reward/loss. Each participant completed 54 trials of potential "gain" or "loss" and had their total earnings summed upon completion. The number of 
risky choices in the gain domain and number of risky choices in the loss domain were used as indicators for the DM latent variable.

\section{Statistical Analyses}

All analyses were conducted in either SPSS v.23. or MPlus v.8.2 (Muthen \& Muthen, 2000-2012). Analyses consisted of examining associations between cannabis use trajectories and anxiety, as well as, the moderating role of anxiety on the relationship between cannabis pattern (i.e., class trajectory) and decision-making performance at T5.

\section{Participant Characteristics.}

Demographics and sample data characteristics were evaluated for normality, skew, kurtosis, and outliers. Additionaly, baseline characteristics revealed group differences for age, sex, race, and amount of cannabis use.

\section{Data Analysis Plan for Aim 1.}

Cannabis use trajectories were estimated to identify unique cannabis use patterns within our sample. Lifetime cannabis frequency was phase to age converted and modeled within an accelerated cohort design. One advantage of utilizing this modeling framework is that it allows for estimation of a trajectory over a longer time-frame than would have been otherwise possible than if it modeled as assessment timepoints (Galbraith, Bowden, \& Mander, 2017). Additionally, this method accounts for planned missingness associated with participant drop-out and missed assessment visits (Galbraith et al., 2017), both of which are common in longitudinal studies. To determine a participant's cannabis use trajectory membership, probabilities were generated and used to develop a model for latent class growth analysis (LCGA) in Mplus using maximum likelihood estimates. A series of models were tested to determine if cannabis growth patterns were best estimated 
as either linear or quadratic (i.e., slopes) growth factors. To determine the most optimal number of classes we selected a model based on theoretical interpretation, parsimony, and fit indices.

After selecting the best fitting model, we implemented maximum probability assignment (MPA) (i.e., hard classification) to identify latent classes of cannabis use trajectories in Mplus. Our decision to use MPA over the statistically novel "three-step approach" for modeling auxiliary variables (Asparouhov \& Muthen, 2014) was guided by findings from our initial trajectory model. When using the three-step procedure, cannabis use trajectory estimates are calculated without a distal outcome (dependent variable) included in the model, whereas MPA includes all outcome variables within the initial model. The three-step procedure statistically accounts for classification uncertainty in an individuals' trajectory group assignment by using class membership probabilities, which leads to reduced bias in model parameter estimates and standard errors when contrasted with other commonly used approaches (e.g., MPA). However, this approach is only particularly helpful when there is a large uncertainty for classifying participants. In other words, the three-step approach is superior when classification certainty is poor (entropy = $<.60)$. However, when class accuracy is high, the MPA approach has comparable efficiency (Asparouhov \& Muthen, 2014). Because entropy (i.e., predictability in class accuracy) was high within our initial model (entropy $=.93$ ), we decided against the threestep approach. Furthermore, the three-step approach is a more complex statistical model that makes it more difficult to incorporate other parts compared to MPA. Next, the subsequent sets of analyses examined (1) cannabis use trajectory group membership as a predictor of changes in anxiety symptomology across the duration of the study (2 years). 
When examined as a distal outcome variable, DASS-21 scores were treated as having unequal means and variances across the cannabis use trajectory groups. These analyses accounted for covariate effects (e.g., participant demographics, depression, baseline alcohol and nicotine use). Additionally, residualized change scores were calculated for DASS-21 symptoms from baseline to T5 to examine change in anxiety across a two-year time-frame. We used a residualized change approach to examine change in anxiety symptomology because it takes into account baseline anxiety levels and additional predictors of interest when examining change over time. That is, within the regression model, anxiety at T5 is regressed on itself at baseline with covariates. Thus, the outcome (residualized) scores represent only variability that is unexplained by baseline levels and is interpreted as change over the study period (Castro-Schilo \& Grimm, 2018). We subsequently followed the same prescribed procedure, to examine the associations between changes in panic disorder and generalized anxiety disorder symptomology from baseline to T5, utilizing data from the C-DISC with cannabis use trajectory membership (see Figures 6-8).

\section{Data Analysis Plan for Aim 2.}

Logistic regressions were conducted to examine differences in the odds of anxiety disorder development between cannabis trajectory groups. Odds ratios provide relative odds of a participant belonging to a cannabis trajectory, per 1-unit increase in disorder outcome. That is, is the development of an anxiety disorder throughout the study period associated with distinct cannabis use trajectories? We ran separate logistic regression models for GAD and PD, using presence of past year disorder as captured by the CDISC, at T3 and T5 assessments. The CDISC predictor variables were binary with " 0 " 
indicating not having the disorder, and " 1 " having the disorder. In all analyses we controlled for sex, and comorbid lifetime alcohol and nicotine frequency. Follow-up analyses explored controlling for depression and exploring sex effects.

\section{Data Analysis Plan for Aim 3.}

First, we identified theoretically relevant covariates that may influence associations between cannabis use and neurocognitive functioning, which included sex, age, estimated IQ, as well as alcohol and nicotine frequency at T5. Next, we generated the DM latent variable, which consisted of the reverse scored Net Total on the Iowa Gambling Task, total number of risky choices on the Game of Dice, and total of risky choices in gain domain and total risky choices in loss domain on the Cups Task at T5, with all indices loading significantly onto the latent factor $(p s<.05)$. We then examined associations between DM T5 performance and cannabis use trajectories, as well as, pastweek anxiety symptoms utilizing the DASS-21 anxiety sub-scale as a moderator for the aforementioned association. To do this, we first ran separate linear regressions model to examine univariate associations between cannabis use trajectory and DM, and between anxiety and DM. Next, to explore the potential moderating influence of anxiety, we then ran a multiple regression which included anxiety, cannabis trajectory, and their interaction. To further explore significant interactions, we conducted separate linear regressions examining the effects various levels of anxiety had on associations between cannabis use trajectories and DM. 


\section{Missing Data Analyses.}

The proportion of participants with complete data at their baseline evaluation was $401(100 \%)$. Cannabis use data collected at the follow-up T2 assessment was available for $391(98 \%)$ participants, followed by data from 383 adolescents $(96 \%)$ for the T3 assessment, 380 adolescents (95\%) at T4, and 387 (97\%) participants at T5. Patterns of missingness were assessed, and we found no evidence that our data was not missing at random (MAR), with no variable differences found for participants with complete versus incomplete datasets. Full-information maximum likelihood (FIML) methodology was applied to all analyses, as the FIML procedure uses all available data points to construct parameter estimates without imputing individual values (Enders \& Bandalos, 2001). All 401 subjects had at least one data point and were therefore used to inform the maximum likelihood estimates.

\section{CHAPTER IV.}

RESULTS

\section{Descriptive Statistics.}

Participants were predominantly high school students of Hispanic ethnicity and White race, with roughly equal representation of males and females. It is to be noted, substance use variables were skewed, as is typical of studies with substance using samples. Although participants reported a varied range of alcohol, nicotine, and cannabis use, use of cannabis generally exceeded use of alcohol and nicotine. At baseline, nine participants met criteria for current cannabis use dependence and no participants met criteria for dependence for any other substance. Median lifetime days of cannabis use at T1 for the whole sample were 21, with interquartile range from one to 145 days. 
Furthermore, few participants met diagnostic criteria for an anxiety disorder at baseline $(\mathrm{n}=17 ; \sim 4 \%)$. Detailed information for primary study variables is presented in Tables $1-4$ by cannabis use trajectory.

\section{Aim 1 Result Part A: LCGA of Cannabis Use Trajectories.}

Initial examination of the unconditional growth model of cannabis use showed acceptable model fit $\left(\chi_{2}[25]=139.95, p<.001 ; \mathrm{CFI}=.91 ; \mathrm{RMSEA}=.10\right)$. The mean estimated intercept $(\bar{x}=.33, S E=0.07)$ and linear slope $(\bar{x}=0.57 ; S E=0.08)$ were significant and positive ( $p$ ’s <.001). Importantly, statistically significant variance estimates were found for the intercept $(p<.001)$ and linear slope $(p<.001)$, indicating significant between-individual variability in initial levels and change over time in cannabis use; that is on average, people increased cannabis use with age but varied in how their use changed over time.

Next a series of LCGA models were conducted to determine the number of latent classes in our sample that would allow us to examine associations between anxiety and different patterns of cannabis use among adolescents.

Following examination of the single-class model, LCGA was used to examine trajectories of cannabis use, controlling for study covariates (e.g., participant demographics, co-occurring alcohol and nicotine use). These analyses were explored in a step-wise fashion, beginning with a two-class solution and ending with a four-class solution. Based on substantive interpretation, parsimony, and fit indices, a three-class model of adolescent cannabis use was identified as providing the best overall solution (see Table 5). Although a four-class model was also considered (see Figure 5), two of these four trajectory groups, both of which were relatively small ( $n$ 's $<35 ;<10 \%$ of total 
401), followed similar escalating patterns of use, which appeared to be largely captured by the more parsimonious, three-class solution. For the three-class model (see Figure 4), the total sample size for each of the classes exceeded $10 \%$ of the total sample, and the trajectories were similar to those observed in prior research (Flory, Lynam, Milich, Leukefeld, \& Clayton, 2004). The three classes were labeled based on frequency of use with the following categories: minimal use (i.e., consistently low levels throughout adolescence), chronic use (i.e., consistently elevated levels throughout adolescence), and escalating use (i.e., significantly accelerating use throughout adolescence). The first class, with 262 participants (65\%), displayed consistently low levels of cannabis use across the study period ("minimal"). For the minimal class, mean estimated intercept $(\bar{x}=$ $3.12, S E=1.28)$ and linear slope $(\bar{x}=1.31 ; S E=0.39)$ were significant in the positive direction $(p$ 's <.001). The second class included 61 participants $(15 \%)$ with chronically high levels of cannabis use, which were characterized by high initial levels of use that remained persistent across assessments ("chronic"). For the chronic class, mean estimated intercept $(\bar{x}=75.97, S E=14.48)$ and linear slope $(\bar{x}=11.76 ; S E=2.97)$ were significant in the positive direction $(p$ 's $<.001)$. Lastly, the third class included 78 participants $(20 \%)$ that displayed initially low levels of cannabis use and escalated their use across the study period ("escalating"). For the escalating class, mean estimated intercept $(\bar{x}=-2.07, S E=$ $7.04)$ and linear slope $(\bar{x}=15.30 ; S E=2.04)$ were significant in the positive direction ( $p$ 's $<.001)$. Additional baseline statistics by cannabis trajectory group are presented in Table 1.

Covariates retained in the analysis included sex, ethnicity, and baseline alcohol and nicotine use. The addition of covariates in the LCGA model did not alter fit statistics, 
cannabis use pattern profiles, or the size of observed classes. Fit information for each model considered can be found in Table 5 and trajectory plots of the three and four class models can be found in Figures 4 and 5.

\section{Aim 1 Results Part B: Cannabis Trajectories and Change in Anxiety.}

Multiple regression models were conducted in Mplus to examine associations between cannabis use trajectory class membership and changes in anxiety symptomology from baseline to two-year follow-up. To identify differential effects of certain anxiety symptoms, models were conducted separately with changes in anxiety symptoms (i.e., in the past week) as measured by the DASS-21, as well as for changes in GAD and PD symptoms as measured by the C-DISC. All models accounted for sex, ethnicity, and comorbid alcohol and nicotine use. The model examining DASS-21 symptoms, found a significant effect of cannabis use trajectory on residualized change in past week anxiety symptoms ( $\beta=.11, \mathrm{p}=.002)$. To explore between group comparisons, we used the grouping command in Mplus. Results from this model revealed a significant betweenclass difference in change in anxiety over time, specifically between the escalating and minimal trajectories $(\beta=-0.322, p<.01)$; see Figure 6 . These findings indicate that participants in the escalating trajectory had larger gains in past week anxiety symptoms across the study compared to the other trajectories. Additionally, associations between sex and changes in past week anxiety symptoms were found across all cannabis use trajectories $(p s<.001)$. We conducted follow-up analyses to explore potential sex differences and created a sex by change in anxiety interaction term and included this term as a predictor in our subsequent models. These follow up analyses revealed that there was a significant interactive effect for change in anxiety and sex on group trajectory $(\beta=.28$, 
$p=.024)$. Further these results revealed that females across all trajectories had greater change (i.e., decreases) in anxiety when compared to males from T1 to T5. Furthermore, the association between cannabis use trajectory membership and change in anxiety among females was significant for both the escalating and chronic trajectories relative to minimal users ( $p s<.05)$, suggesting that frequent and increasing cannabis use impacts anxiety more among adolescent females when compared to males. However, the main finding of sex diminished for males ( $p s>.05$ ), indicating change in anxiety does not vary for males across trajectory groups. Thus, it appears that being female accounts for all sex differences in anxiety change and cannabis use group.

The same prescribed procedure above was conducted with change scores in GAD and PD symptoms; however, no significant associations were found between cannabis group membership and change in these symptoms and (ps>.05); see Table 6 and Figures 7 and 8.

\section{Aim 2 Results: Odds of Developing an Anxiety Disorder at T3 or T5.}

To determine if cannabis use trajectory predicted anxiety disorder development, logistic regressions were conducted with group-wise comparisons (i.e., chronic vs. minimal; chronic vs. escalating; minimal vs. escalating) are provided in Table 7.

Development of PD at T3:

After accounting for sex $(p<.001)$, alcohol $(p=.009)$, and nicotine ( $p=.002)$ use, there was no effect of cannabis use trajectory group on the odds of developing PD by the one-year follow-up assessment (i.e., T3); $(\beta=.877, p>.05)$; see Figure 7. Odds ratios for group wise comparisons, revealed minimal users were more at risk of developing PD $(p<.05)$. No other group comparisons were significant $(p>.05)$. However, due to the 
observed association of sex with PD symptoms, we conducted follow-up testing with a sex by cannabis group interaction term which revealed a significant interaction for sex and cannabis use group on development of PD at T3 $(\beta=.773, p<.001)$. To further explore sex differences, we conducted our initial model separately for males and females, and a significant association was found between cannabis use class and PD development for females $(p=.002)$, but not males $(p=.610)$. Such that females across all cannabis trajectories had greater likelihood of developing PD than males.

\section{Development of PD at T5:}

After accounting for sex $(p<.001)$, alcohol $(p=.005)$, and nicotine $(p=.001)$ use, cannabis use trajectory class membership did not predict the odds of developing PD by the two-year follow-up assessment $(\mathrm{T} 5 ; p>.05)$. Nor, did the group comparisons between minimal and escalating trajectories reveal significant differences in PD development ( $p$ $>$.05). Additionally, odds ratio comparisons between groups with the chronic cannabis use trajectory could not be conducted because no members within this group developed PD at T5. However, due to the observed association of sex with PD symptoms, we once again conducted follow up testing with an interaction term of sex by cannabis group. Within these analyses, after accounting for the previously mentioned covariates, there was a significant interaction effect for sex by cannabis group $(\beta=.713, p<.001)$. To explore sex differences between groups, we ran our initial model separately for males and females, and a significant association was found on development of PD at T5 for males ( $\beta$ $=-.915, p<.001)$. This effect indicated that males in the escalating trajectory had significantly higher odds of developing PD than males in other groups. 


\section{Development of GAD at T3:}

After accounting for sex $(p<.001)$, alcohol $(p=.004)$, and nicotine $(p=.001)$ use, an association was found between cannabis use trajectory class membership and GAD development by the one- year follow up assessment (T3; $p=.05)$, see Figure 8 . Follow up analyses revealed a significant difference in development of GAD development between the minimal trajectory and the escalating trajectory $(\beta=.667, p<.001)$, such that participants in the minimal group had significantly greater odds of developing GAD than those participants who were increasing their cannabis use across a one-year period. There was also a significant difference in developing GAD between the chronic and escalating trajectories at the one-year follow-up $(\beta=-.499, p<.001)$. However, odds ratio values for group comparison could not be computed with participants from the chronic trajectory because no members developed GAD. Yet, these findings indicate that adolescents with consistently high levels of use had less risk of developing GAD than those exposed to minimal or increased use.

Additionally, because there is accumulating evidence to suggest that internalizing disorders develop more in females and males, we wanted to explore potential sex effects among our cannabis use trajectories. Since sex was significantly related to cannabis use trajectory and GAD development in our sample, we conducted follow up analyses with a sex by cannabis group interaction term to explore potential sex effects. Within these follow up analyses, after accounting for the previously mentioned covariates, a significant association was found for cannabis use class membership and sex on GAD development for females $(p<.001)$ when compared to males $(p=.610)$. To identify sex differences between classes, further analyses were employed. The results of these 
analyses revealed females within the minimal trajectories were more likely to develop GAD when compared to males in this group $(p<.05)$.

Development of GAD at T5:

After accounting for sex $(p<.001)$, ethnicity $(p=.022)$, alcohol $(p=.005)$, and nicotine $(p=.001)$ use, no association was found between cannabis use trajectory class membership and GAD development at the two-year follow up assessment (T5; $p>.05)$. Comparable to GAD T3 results, odds ratio values for group comparison could not be computed with participants from the chronic trajectory because no members developed GAD. However, due to the significant independent effects of sex and cannabis group on this association at T3, we also employed these same follow up models to explore interactive effects of sex and cannabis group on T5 GAD developments. Within these follow-up analyses, after accounting for the previously mentioned covariates, a significant association was found for cannabis use class membership and GAD development for females $(p=.004)$ and trending towards significance for males $(p=.053)$ at two-year follow up. Further exploration of sex differences revealed, a greater GAD development among female users in the escalating trajectory $(\beta=-.830, p=.004)$ when compared to those in the chronic and minimal trajectories. These results indicate that females in the escalating trajectory had greater risk of developing GAD than females with other patterns of cannabis use.

\section{Accounting for Depression with PD \& GAD Development:}

As a result of frequent comorbidity of depression and anxiety pathology, all analyses were repeated accounting for depression at baseline and two-year follow up. Due to relatively minimal clinical levels of anxiety within our sample, we refrained from 
including depression in our initial models because of potentially over-accounting for variance captured by comorbid internalizing disorders. Nevertheless, across all follow-up analyses all main findings remained unchanged at both T3 and T5 for PD and GAD development.

\section{Aim 3 Results: Anxiety as a Moderator of the Relationship Between Cannabis Use Trajectories and Decision-Making.}

At T5, there was no association found between cannabis use trajectory predicting decision making $(p>.05)$. However, there was a significant main effect of anxiety $(\beta=-$ $.30, p=.035$ ) on DM indicating that less anxiety was associated with better (i.e., less risky) DM. Next, to determine if the association between cannabis trajectory membership and DM varied by anxiety, we included an anxiety by cannabis group interaction term and found a significant interaction $(\beta=.237, p=.032)$, see Figure 10 . To explore the interaction, results from follow up testing revealed that anxiety moderates the relationship between cannabis group trajectory and decision-making among adolescents only in the minimal trajectory $(\beta=-.185, p=.042)$, but not among those within the escalating or chronic trajectories $(p>.05)$. These findings suggest that anxiety levels impact DM performance to a greater extent among participants with less frequent cannabis use than among those with heavier use, see Table 8. Additionally, these results suggest that greater anxiety among minimal users is associated with less risky decision-making. However, it appears that that levels of anxiety do not influence the relationship between cannabis use trajectories and risk-taking among those who escalate in use or are chronic users.

We used the same procedure described in previous aims to control for the potential influence of depression on the associations between anxiety, cannabis group, 
and DM. These analyses revealed that the main effect of anxiety on DM dissipated ( $p$ $=.463)$, yet the interaction term of anxiety*cannabis group remained $(\beta=.216, p=.039)$. Furthermore, the main effect of depression on DM was marginally significant across all cannabis groups $(\beta=-.119, p=.057)$, suggesting that DM may be better (i.e., less risky) at higher levels of depression for teens within all cannabis trajectory groups. These findings suggest that depression may influence the association between anxiety and cannabis trajectory on DM. To further explore support for a cross-over interaction, such that the effect of anxiety on the association between cannabis group and DM depends on the level of depression between groups, we conducted the same set of models with the addition of an anxiety*depression interaction term. A significant interaction of anxiety*depression term was found $(\beta=-.15, p=.050)$. However, this finding was revealed only for the escalating trajectory, and suggests that both depression and anxiety interact and is related to poorer DM among users who increase their cannabis use rather than those with minimal or steady chronic use.

\section{CHAPTER V.}

\section{DISCUSSION}

This set of studies examined associations between anxiety and cannabis use trajectories with a sample of adolescents at risk for escalation in cannabis use. Findings revealed that distinct patterns of adolescent cannabis use were associated with different anxiety-related outcomes. 


\begin{abstract}
Aim 1
Results from our Aim 1 analyses found that, on average, anxiety decreased over the course of the two-year study period. We identified three main trajectories of cannabis use in our sample: minimal use (i.e., consistently low levels throughout adolescence), chronic use (i.e., consistently elevated levels throughout adolescence), and escalating use (i.e., significantly accelerating use throughout adolescence). When examining associations between cannabis use trajectory and changes in anxiety, results revealed that adolescents in the escalating trajectory of cannabis use showed greater change in anxiety symptoms over two years when compared to minimal and chronic users. Specifically, adolescents in the escalating trajectory showed lesser decreases in anxiety over time when compared to those in the minimal use trajectory. This suggests that increasing and frequent cannabis use during adolescence may not an effective strategy for managing anxiety and potentially leads to higher levels of anxiety relative to peers with different patterns of use.
\end{abstract}

These results may be accounted for, at least in part, by several factors. First, the expression of endocannabinoid CB1 receptors across the brain follows an age-dependent downregulation (i.e., decreases in receptor numbers) which is associated with neural structure development (Heng, Beverley, Steiner, Tseng, 2011). During adolescence, the largest changes in CB1 expression occurs first in limbic regions and the prefrontal cortex (Ellgren et al., 2008; Heng et al., 2011), regions of the brain which are associated with emotion, anxiety, and decision-making. In addition to the natural course of cannabinoid receptor reduction, prior work has found that short- and long-term use of cannabis during adolescence contributes to neurochemical changes and subsequent deviations in 
cannabinoid receptor densities, most notably among CB1 receptors (Dalton \& Zavitsanou, 2010). Since, structures of the brain and the endocannabinoid system are undergoing developmental changes in adolescence, it is possible that since THC binds to CB1 receptors, neurocircuits may be affected and contribute to psychological symptoms (Bossong \& Niesink, 2010).

In addition to alterations in receptor densities, adolescent cannabis use has been associated with desensitization of CB1 receptors, which is a reduction in receptormediated G-protein-coupled activity (Burston, Wiley, Craig, Selley, \& Sim-Selley, 2010). Attenuation of receptor G-protein activity in neural receptors are associated with variations in the regulation of physiological processes and neurotransmission (Howlett et al., 2004). Within our study, we accounted for age by utilizing an accelerated cohort design to generate our cannabis trajectories, and thus accounted for variance associated with age as part of our models. However, different neural effects may have occurred between cannabis groups, especially among users in the chronic and escalating trajectories. That is, heavier patterns of use may have impacted the downregulation of CB1 receptors, differently between groups, leading to differences in CB1 expression. These possible differences in receptor expression subsequently may have influenced growth in psychopathology and anxiety levels.

Another plausible explanation is that adolescents within our sample were selfmedicating with cannabis, consistent with the idea that increased substance use is a result of maladaptively coping with emotional difficulties (Bolton, Robinson \& Sareen, 2009; Khantzian, 1997; Turner, Mota, Bolton, \& Sareen, 2018). As we highlighted earlier, prior research has shown support for a dose-biphasic effect with amount of THC consumed; 
such that initial low levels are often associated with anxiolytic effects, whereas chronic and heavy use is associated with increased anxiogenic symptoms (Rey et al., 2012). Considering that the anxiety effects we observed are associated with amount of cannabis consumed, this may explain why participants within the escalating trajectory are showing the greatest change in anxiety symptomology during the study period. Further, participants within the escalating trajectory are showing a slower decrease in anxiety when compared to other groups. Interestingly, we did not find significant differences between chronic and minimal patterns of cannabis use on changes in anxiety symptoms. We attribute these findings, in part, to additional factors within the biopsychosocial framework of addiction. For example, adolescents who engage in initially high levels of cannabis use and maintain this pattern overtime are not changing their use on the basis of psychological or somatic symptoms of anxiety that may develop over time, indicating they may have more appropriate anxiety-regulating strategies. However, more chronic levels of early use may be influenced by genetic susceptibilities to addiction. Whereas, escalating users may initiate use first following more social and psychological pressures, and change their use over time to adapt with poor social relations and emotional dysregulation.

\section{$\operatorname{Aim} 2$}

To determine if distinct cannabis use trajectories were associated with development of PD or GAD across the study period several models were conducted and evaluated. Our findings revealed that adolescents among all cannabis use trajectories did not differ in their development of PD during the course of the study (i.e., T3 or T5). However, development of GAD at T3 was significantly associated with cannabis use 
group but not at T5. These results are somewhat surprising since we hypothesized that increased cannabis use would be associated with more clinically elevated levels of anxiety. Our hypothesis was guided by prior research in both rodent and human samples that have indicated that the desensitization of $\mathrm{CB} 1$ receptors in limbic regions by cannabis use is related to significantly elevated levels of anxiety (Hirvonen et al., 2012; O'shea et al., 2004). Remarkably, our Aim 1 findings are consistent to these results, in which we found escalating users to have slower decreases in anxiety, translating to potentially higher levels of anxiety symptoms over time when compared to minimal users. It may be that the teens in the minimal trajectory, who have clinically elevated symptoms of anxiety, have other contributing factors that may better account for their anxiety than solely cannabis use. This is of relevance since our follow-up findings found interactive effects with cannabis group and sex on anxiety disorder development. Within our sample, females were more likely to develop PD or GAD when compared to males and is in agreement with prior research that has found anxiety to be more common among females than males (Altemus, 2006).

Furthermore, developmental considerations between participants in each trajectory may have contributed to our Aim 2 findings, in which participants in the minimal group had greater odds of developing GAD than adolescents in the escalating trajectory. Although we recruited a sample with comparable demographics and substance use histories, it is possible that a subset of individuals may have skewed anxiety outcomes differently across our three cannabis use trajectories due to unknown variables not assessed. 
Additionally, as previously highlighted, anxiety follows a natural waxing and waning pattern across adolescence. Prior research has found differences between the rate of growth and reduction of PD and GAD symptom throughout the adolescent developmental period, such that PD symptoms decrease in early to middle adolescence compared with increases in GAD symptomology (Hale III et al., 2008). Correspondingly, adolescent depressive symptoms are associated with future onset of GAD development (Fergusson \& Woodward, 2002; Moffit et al., 2007), and evaluation of depression onset or evaluating GAD across a larger time-frame may have provided alternative outcomes. In addition, several other factors are considered that may have contributed to our results. When examining mean group differences of PD and GAD symptoms at T1, a significant difference in GAD symptoms was observed, specifically greater GAD symptoms were present among participants in the minimal trajectory when compared to those in the escalating trajectory. Although these symptoms were not of clinical anxiety severity at $\mathrm{T} 1$ yet, it does support the possibility that those within the minimal trajectory joined our study as more anxious individuals and which could have influenced anxiety growth to clinically elevated levels. Furthermore, symptoms of worries about the future, health, and illness, are notable symptoms of GAD (Newman, Llera, Erickson, Przeorski, \& Castonguay, 2013). Potential lack of worries associated with the health risks of smoking could have served as a protective factor for more chronic and increasing levels of cannabis use (Wells \& Carter, 1999) among our participants. Alternatively, within the early stages of use cannabis may be adaptive for managing acute physiological symptoms of anxiety (as evidenced by our lack of PD development differences between cannabis groups) but determining whether these results are evident across larger windows of time 
or are permanent warrants further examination. Thus, it appears the relationship between cannabis use and anxiety disorder development share a bi-directional association with one another yet identifying causal factors for poorer clinical outcomes remains challenging.

\section{$\operatorname{Aim} 3$}

Upon examining whether anxiety moderated the relationship between cannabis use trajectories and risky DM, our results did not find evidence for a main effect of cannabis pattern on DM. However, our results revealed a main effect for anxiety, as well as a significant interaction between patterns of cannabis use and anxiety, indicating that cannabis use patterns are associated with DM differently at various levels of anxiety. Our initial findings suggest that minimal users with lower levels of anxiety had better DM (i.e., less risk-taking) than those with higher levels of anxiety. These findings lend support to prior evidence that have found increased anxiety is associated with risk averse behaviors (Lorian \& Grisham, 2011; Maner \& Schmidt, 2006). Yet, it is interesting to note that this established association is not observed (i.e., disrupted) at escalating and more chronic levels of cannabis use. One potential hypothesis for our lack of risk avoidance with higher levels of anxiety in escalating and chronic users may be related to changes in brain executive functioning and emotional processing brain regions. As previously discussed, THC binds to receptors in these areas of the brain (e.g., prefrontal cortex, limbic area), and heavy prolonged use may disrupt neural pathways and signaling that alter the association between anxiety and DM, ultimately resulting in a propensity for making risky decisions, regardless of anxiety. However, we did not find a main effect for cannabis trajectory on DM. 
Interestingly, however, these associations between anxiety, cannabis use patterns, and DM appear to be influenced by comorbid depression. Exploratory, follow-up analyses indicated that increased levels of depression interacted with anxiety to influence greater risk-taking among adolescents who "escalate" in use. Thus, it appears that associations between increasing cannabis use and anxiety vary as a function of depression among adolescents who increase their use over time. Although longitudinal research suggests that anxiety typically develops before depression in most cases (Feng, Shaw, \& Silk, 2008), and is more common than depression (Axelson \& Birmaher, 2001), it appears risky DM may be influenced by interactive effects of anxiety, depression, and cannabis use.

Furthermore, although sex had significant associations with anxiety, cannabis group, and DM performance, the stability of this relationship fluctuated as a result of accounting for depression. That is, the main effect for sex between group comparisons became insignificant when depression was added into our models. Nonetheless, females' DM performance was more adversely affected by anxiety and depression across cannabis groups, suggesting that cannabis and internalizing pathology interact differently for males and females. These sex effects map onto prior research that has indicated internalizing pathology is more common among adolescent females than males (Soloman \& Herman, 2009), and can lead to poorer neurocognitive outcomes (Eysenck, Derakshan, Santos, \& Calvo, 2007). Accordingly, although we did not find evidence to support prior claims that cannabis use affects DM (Bolla, Eldreth, Matochik, \& Cadet, 2004), our results replicate studies who have not found effects of cannabis on DM (De Bellis et al., 2013; Eldreth, Matochek, Cadet, \& Bolla, 2004; Gonzalez et al., 2012; Lyons et al., 2004), including a 
preliminary study from our lab utilizing the same sample with data from baseline through T3 (Duperrouzel et al., 2019).

Our results also complement the extant literature and indicate that female users may be at a greater risk than males for adverse mental health consequences and cognitive outcomes as a result of adolescent cannabis use, regardless of cannabis use trajectory.

\section{Strengths of Present Study.}

There are several strengths of the present study. First, the study followed a relatively large sample of youths at risk for cannabis use escalation with an impressive retention record across two years. Across the study windows, multiple data points regarding demographic, social, emotional, substance use, and cognitive functioning were captured and allowed for the opportunity to evaluate change across these domains. Additionally, previously identified patterns of cannabis use were replicated within our sample and included steady low levels, chronic high levels, and an increasing pattern of cannabis use. Overall, the wealth of data collected across five time-points allowed for further examination of associations between patterns of cannabis use and adverse anxiety outcomes than currently available in the literature. Although one of the most frequent reasons cited for cannabis use among teens is for the reduction of stress, teasing apart anxiety by disorder sub-types further enhances our understanding on the complex relationships between cannabis use and anxiety. Our study was also able to identify differences in the severity of anxiety sub-types and identifying which patterns of use are more susceptible to developing clinical levels of GAD and PD over time. Interestingly, patterns of cannabis use appear to affect differences in anxiety over time. Additionally, our results suggest that levels of anxiety can moderate relationships between cannabis use 
and executive functioning (e.g., decision making). Thus, from our findings, it appears that cannabis use and anxiety have a bi-directional influence that impacts growth within each domain over time throughout mid to late adolescence.

Within our follow up analyses, we also identified several interesting avenues for future research which included the identification of sex differences in these associations, as well as the influence of comorbid depression. Through these findings, we have been able to further identify individuals who may be most at risk for poorer anxiety outcomes, with our results suggesting females and individuals with comorbid depressive symptoms to be most affected by higher levels of cannabis use. Interestingly, our study was also able to shed light on the potential neurocognitive effects of anxiety and cannabis use on risky DM. Although adolescence in general is often characterized as a period of heightened risk-taking (Casey, Getz, \& Galavan, 2008), factors that contribute to even riskier DM have the potential to lead to extreme consequences such as teenage pregnancy, school underachievement and dropout, other substance use, and even death (Chakravarthy, Shah, \& Lotfipour, 2013). Our findings suggest that cannabis use patterns do not predict decision making outcomes among youth in the early stages of use. However, results from our model looking at bidirectional influences of cannabis use trajectory and anxiety on decision-making suggest the anxiety moderates the relationship between cannabis and risk-taking. More specifically, our findings identified that a minimal cannabis use pattern and greater levels of anxiety can interact to make DM better (i.e., less risky). Interestingly, we did not find statistical evidence that among teens with higher levels of anxiety, heavier patterns (i.e., chronic and escalating trajctories) were associated with risker DM. However, when representing the anxiety by cannabis group 
interaction data in a graph format, it appears that the relationships between heavier patterns of CU and DM are affected differently at various levels of anxiety, when compared to teens with minimal cannabis use. Thus, continuing to identify factors that contribute to more negative outcomes can help aid in targeted prevention and intervention efforts. It will be important to continue to examine these associations between levels of anxiety and patterns of $\mathrm{CU}$ on DM over time to determine if these associations fluctuate, dissipate, or are stable.

Furthermore, our results partially support the self-medication hypothesis regarding somatic symptoms of anxiety (as indicated by Aim 1 findings), which posits that substance use is initiated/maintained in order to relieve uncomfortable affective states, such as anxiety or depression (Khantzian, 1997). Adopting a self-medicating motive for cannabis use as a youth can help maintain maladaptive coping mechanisms, and ultimately lead an individual to the development of substance use disorders and other addictions. While substances like cannabis may be perceived as less harmful when compared to other drug classes such as opiates and amphetamines (Miech et al., 2019), using a substance in an effort to regulate emotional states through drug related "highs" and "euphoria" rather than addressing maladaptive stress, anxiety, and depressive contributes to negative reinforcement cycles (Koob \& Kreek, 2007; Sinha, 2008). Lastly, these findings extend and support prior findings from our lab, in which higher levels of cannabis use appear to be most associated with more negative psychological consequences. Although we did find evidence for greater probability of the minimal trajectory developing GAD when compared to chronic and escalating users, we suspect that these results may be transient and not indicative of long-term negative outcomes of 
low use, or may be better accounted for by our participant sampling methods.

Nonetheless, we suspect that the lack of evidence for more adverse anxiety outcomes among the escalating and chronic trajectories may also be heavily influenced by the relatively minimal levels of reported anxiety within our study.

\section{Limitations.}

These findings should be considered in light of several limitations. First, our sample consisted of adolescents in the early stages of cannabis use in an attempt to capture different growth patterns of use over a two-year period. However, previous studies examining cannabis use trajectories across different developmental stages have found that patterns of use vary depending on the age of participants captured. For example, a study examining a large community sample of high-school students $(n=1,205)$ found five distinct cannabis patterns emerge across their sample, including our aforementioned trajectories, as well as, decreasers and experimental users (Windle \& Wiesner, 2004). On the other hand, a large regional study, recruited 5,833 participants from California and Oregon with ages ranging between 13-23 and found four distinct patterns of cannabis use over a 10-year period (Ellickson et al., 2004). Since the present study recruited a robust sample from the greater Miami area, when comparing the sample size to other studies examining cannabis use trajectories, our findings remain somewhat disparate. With an even larger study sample, we potentially could have identified different cannabis use trajectories than the ones we captured. Additionally, within our study, we were interested in utilizing LCGA to identify patterns of use within our sample specifically (i.e., teens in early stages of cannabis use), and not necessarily indicative of cannabis patterns found in the population at large. Although we utilized an accelerated 
cohort design to capture a larger range of ages within our sample, the estimates for these simulated ages were influenced by the participant characteristics of our sample (e.g., majority exposed to alcohol, nicotine, or cannabis use, even if only minimally). Perhaps with a more diverse sample, we would have identified different trajectories, with possibly initiation of heavy use at different ages. Furthermore, if we had studied our participants for longer, and even across different developmental periods, such as young adulthood, we may have been able to identify trajectories like adolescent-limited trajectories or even "decreasers," that is individuals who decrease use over time. Thus, it is possible that our results depict only a small snapshot of the associations between adolescent cannabis use and anxiety, and that a larger more diverse sample of adolescents may have had different class trajectories and anxiety outcomes.

Other study limitations are related to the characteristics of our sample and parent study design. For instance, approximately $90 \%$ of participants in our sample were of Hispanic/Latino/a ethnicity. According to the MTFS, Hispanic adolescents report earlier initiation of cannabis use and greater use during adolescence when compared to Caucasian adolescents (Johnston et al., 2018; Miech et al., 2015). Hence, the findings we report here may not generalize to larger more diverse samples that include individuals from a more varied range of ethnic backgrounds. Additionally, parent study exclusion criteria included clinically elevated levels of psychopathology in several areas at the time of screening (e.g. mood disorders, thought disorders, etc). Nevertheless, $7 \%$ of our participants did meet criteria for internalizing disorders at baseline, and an additional 8\% developed it over the course of the study. Because our main outcome variables were related to more elevated levels of anxiety, our results should be interpreted within the 
framework of a typically developing healthy sample. Associations between cannabis use, anxiety, and decision-making may vary among individuals with more severe and clinically elevated levels of anxiety.

It is also possible that the tools employed to assess anxiety and mental health may not have been the optimal. For instance, the C-DISC used criteria from the DSM, 4 th edition revised. However, when the DSM-5 was published in May 2013, criteria for PD changed. Of the main noteworthy changes, PD and agoraphobia (i.e., the fear of situations or places that cause panic), were separated, and changes were made to panic attack types. Additionally, PD diagnosis would not occur if it is better accounted for by another disorder such as SAD (Asmundson, Taylor, \& Smits, 2014). Since, our study did not capture SAD symptoms, it is difficult to determine whether panic attacks were associated with SAD or true PD. Furthermore, because we did not examine SAD, replication of prior established effects with CU was restricted with our sample.

Moreover, our findings reported on the associations between cannabis trajectories and changes in PD and GAD symptoms, whereas the extant literature has focused more heavily on associations between changes in adolescent cannabis use and symptom changes in $\mathrm{SAD}$. As $\mathrm{SAD}$ is the most highly prevalent anxiety disorder among cannabis users, and a proposed risk factor for cannabis dependence (Buckner et al., 2008), it would have been informative to replicate these findings in our sample. Additionally, because our follow-up analyses indicated sex differences between associations with anxiety and cannabis trajectory group, most notably among females, it is contrasting to sex differences associated with SAD and cannabis use; in which males are more adversely affected than females (Buckner, Zvolensky, \& Schmidt, 2012). Despite the reliability and 
validity of the measures used to assess psychopathology in our sample of adolescents, future work would benefit from including multiple measures of anxiety that capture more common adolescent anxiety disorders to replicate and extend previous findings. Other measures that would be helpful in the assessment of adolescent anxiety with cannabis use would be to account for level of neuroticism with sample participants.

Finally, previous work suggests that the association between SAD and adolescent cannabis use is related to peer relations and social support (Nelemans et al., 2016). Earlier research indicates that adolescents with greater peer involvement may have more opportunities to participate in experimentation and cannabis abuse. Whereas, teens with SAD may be more socially isolated. Since adolescent cannabis use generally occurs with peers, those not participating in social engagements with peers may be limited in their opportunities to use cannabis routinely (Nelemans et al., 2016). It is possible that our results may also have been influenced by social factors we did not explore. Consequently, more work is needed to determine how and what social mechanisms contribute to adverse mental health and cognitive outcomes among cannabis using youth.

\section{Conclusions and Future Directions.}

In conclusion, our findings contribute to the understanding of the complex associations between adolescent cannabis use and anxiety. It appears that escalating pattern of cannabis use is worse for current anxiety symptoms (i.e., past week) among teens. Our analyses specifically found that increasing use across the study period was associated with higher levels of past week anxiety symptoms at two year follow up than when compared to minimal and chronic patterns of cannabis use. Alternatively, it appears that adolescents with the lowest levels of cannabis use have the greatest risk of 
developing an anxiety disorder. We highlighted several hypotheses as to why this may occur, and include contributing factors of SAD not examined here, heightened initial levels of anxiety, and other external factors not evaluated (e.g., neuroticism). Lastly, we found that various levels of anxiety interacts with different patterns of cannabis use to influence DM differently, and that this association also varies with depression and sex. These findings are of great importance because it helps identify adolescents who may potentially be at risk for more adverse neurocognitive outcomes in the future. Although further work is needed to solidify our understanding of these relationships, our results indicate that adolescent cannabis use is associated with changes in anxiety, anxiety disorder development, and interacts with anxiety to influence risky decision-making. It is also evident that distinctive patterns of cannabis use affect males and females differently and more work is needed to help identify mechanisms that contribute to these disparate outcomes. Furthermore, comorbidity with depressive symptoms appears to influence associations between anxiety and cannabis use, and more work is needed to identify how differences in affective and mood symptoms interact.

The results presented here help further inform clinical decision making for mental health professionals treating adolescents with anxiety disorder symptoms, in regard to anxiety course with comorbid patterns of cannabis use. Furthermore, prevention of clinically elevated anxiety symptoms during early adolescence may have important secondary effects on preventing future cannabis use and adverse DM, which may lead to negative outcomes such as teen pregnancy, school dropout, and other substance use. Mental health professionals aimed at reducing levels of anxiety through methods focused on acquiring healthy coping mechanisms for stress and worries, such as through cognitive 
behavioral therapy (CBT) strategies may subsequently buffer potential cannabis use for self-medicating mechanisms. Additionally, mental health providers should assess comorbid depression among teens who use cannabis and have anxiety, especially among females, as they may engage in more riskier decision-making. Thus, it is important for prevention and intervention efforts to not only be aware of psychopathological and substance use factors contributing to poorer outcomes, but also be aware that other social factors, such as peer involvement, psychological variables like neuroticism, and other biological influences (e.g., genes) may be contributing to these outcomes. Earlier we presented the biopsychosocial model in an attempt to guide our aims and hypotheses in relation to associations between anxiety and cannabis use. Greater targeted prevention for ameliorating anxiety among adolescents should take a systems perspective in an attempt to identify social, psychological, and biological factors contributing to negative outcomes and maladaptive coping mechanisms among adolescents.

Correspondingly, the National Institute of Health's partnership titled, Collaborative Research on Addiction (CRAN), promotes an interdisciplinary framework in which numerous national agencies can receive funding to aid and further our understanding of addiction science. Through a systems approach of evaluation, addiction research will be enriched through collaborations across genetic, epigenetic, molecular, neurobiological, behavioral, and social science disciplines.

Currently, there are several large, longitudinal government-funded projects that are in progress and seek to identify factors that contribute to substance abuse. The Adolescent Brain and Cognitive Development (ABCD) study, is a national landmark project that began in September 2015 and recruited over 11,000 children aged 9 to 10. 
The project is comprised of a consortium of 21 research sites across the United States to recruit a nationally represented sample of children. Participants will complete numerous assessments each year for ten years, in an effort to collect data on social, emotional, biological, cognitive, and neural domains throughout development into young adulthood. Since adolescence is characterized as a period of substance use initiation and heightened developmental changes, understanding factors that contribute to harmful substance use trajectories is critical. Data gathered from participants during the pre-adolescent, adolescent, and post-adolescent years will hopefully allow for precursors of substance abuse to be identified and aid in prevention and intervention efforts. Other current CRAN funded projects include projects examining adolescent alcohol use, drug use prevention among HIV samples, cocaine addiction, and methamphetamine reward and relapse mechanisms. Furthermore, at the time of this dissertation (September 2019), 25 CRAN related research projects had received federal funding, with an additional two pending revision approval. Findings from these studies will further promote our understanding on factors that contribute to more adverse outcomes from substance use. 


\section{References}

Abuse, N. I. on D. (n.d.). Monitoring the Future Survey: High School and Youth Trends. Retrieved April 6, 2017, from https://www.drugabuse.gov/publications/drugfacts/monitoring-future-survey-highschool-youth-trends.

Altemus, M. (2006). Sex differences in depression and anxiety disorders: potential biological determinants. Hormones and behavior, 50(4), 534-538.

American Psychiatric Association. (2013). Diagnostic and statistical manual of mental disorders (5th ed.). Arlington, VA: Author.

Asmundson, G. J., Taylor, S., \& AJ Smits, J. (2014). Panic disorder and agoraphobia: An overview and commentary on DSM-5 changes. Depression and anxiety, 31(6), 480-486.

Asparouhov, T., \& Muthén, B. (2014). Auxiliary variables in mixture modeling: Using the $\mathrm{BCH}$ method in Mplus to estimate a distal outcome model and an arbitrary secondary model. Mplus Web Notes, 21(2), 1-22.

Axelson, D. A., \& Birmaher, B. (2001). Relation between anxiety and depressive disorders in childhood and adolescence. Depression and anxiety, 14(2), 67-78.

Baker, D., Pryce, G., Giovannoni, G., \& Thompson, A. J. (2003). The therapeutic potential of cannabis. The Lancet Neurology, 2(5), 291-298.

Batalla, A., Bhattacharyya, S., Yücel, M., Fusar-Poli, P., Crippa, J. A., Nogué, S., Martin-Santos, R. (2013). Structural and functional imaging studies in chronic cannabis users: a systematic review of adolescent and adult findings. PloS One, 8(2), e55821.

Bechara A (2007). Iowa Gambling Task Professional Manual Lutz, FL: Psychological Assessment Resources

Bechara, A., Damasio, A. R., Damasio, H., \& Anderson, S. W. (1994). Insensitivity to future consequences following damage to human prefrontal cortex. Cognition, 50(1), 7-15.

Beesdo, K., Knappe, S., \& Pine, D. S. (2009). Anxiety and anxiety disorders in children and adolescents: developmental issues and implications for DSM-V. Psychiatric Clinics of North America, 32(3), 483-524.

Blakemore, S. J., \& Robbins, T. W. (2012). Decision-making in the adolescent brain. Nature neuroscience, 15(9), 1184. 
Bolla, K. I., Eldreth, D. A., Matochik, J. A., \& Cadet, J. L. (2005). Neural substrates of faulty decision-making in abstinent marijuana users. NeuroImage, 26(2).

Bolton, J. M., Robinson, J., \& Sareen, J. (2009). Self-medication of mood disorders with alcohol and drugs in the National Epidemiologic Survey on Alcohol and Related Conditions. Journal of affective disorders, 115(3), 367-375.

Bossong, M. G., \& Niesink, R. J. (2010). Adolescent brain maturation, the endogenous cannabinoid system and the neurobiology of cannabis-induced schizophrenia. Progress in neurobiology, 92(3), 370-385.

Braida, D., Limonta, V., Malabarba, L., Zani, A., \& Sala, M. (2007). 5-HT 1A receptors are involved in the anxiolytic effect of $\Delta$ 9-tetrahydrocannabinol and AM 404, the anandamide transport inhibitor, in Sprague-Dawley rats. European Journal of Pharmacology, 555(2), 156-163.

Brand, M., Fujiwara, E., Borsutzky, S., Kalbe, E., Kessler, J., \& Markowitsch, H. J. (2005). Decision-making deficits of korsakoff patients in a new gambling task with explicit rules: Associations with executive functions. Neuropsychology, 19(3), 267277.

Bronfenbrenner, U. (1979). The ecology of human development. Harvard university press.

Bronfenbrenner, U. (2005). Making human beings human: Bioecological perspectives on human development. Sage.

Brook, J. S., Lee, J. Y., Brown, E. N., Finch, S. J., \& Brook, D. W. (2011). Developmental Trajectories of Marijuana Use from Adolescence to Adulthood: Personality and Social Role Outcomes. Psychological Reports, 108(2), 339-357.

Brown TA, Campbell LA, Lehman CL, Grisham JR, Mancill RB. Current and lifetime comorbidity of the DSM-IV anxiety and mood disorders in a large clinical sample. J Abnorm Psychol 2001;110:585-599.

Buckner, J. D., Schmidt, N. B., Bobadilla, L., \& Taylor, J. (2006). Social anxiety and problematic cannabis use: evaluating the moderating role of stress reactivity and perceived coping. Behaviour Research and Therapy, 44(7), 1007-1015.

Buckner, J. D., Bonn-Miller, M. O., Zvolensky, M. J., \& Schmidt, N. B. (2007). Marijuana use motives and social anxiety among marijuana-using young adults. Addictive Behaviors, 32(10), 2238-2252. 
Buckner, J. D., Schmidt, N. B., Lang, A. R., Small, J. W., Schlauch, R. C., \& Lewinsohn, P. M. (2008). Specificity of social anxiety disorder as a risk factor for alcohol and cannabis dependence. Journal of Psychiatric Research, 42(3), 230-239.

Buckner, J. D., Heimberg, R. G., Matthews, R. A., \& Silgado, J. (2012). Marijuanarelated problems and social anxiety: the role of marijuana behaviors in social situations. Psychology of Addictive Behaviors, 26(1), 151.

Buckner, J. D., Heimberg, R. G., Schneier, F. R., Liu, S.-M., Wang, S., \& Blanco, C. (2012). The relationship between cannabis use disorders and social anxiety disorder in the National Epidemiological Study of Alcohol and Related Conditions (NESARC). Drug and Alcohol Dependence, 124(1), 128-134.

Buckner, J. D., Zvolensky, M. J., \& Schmidt, N. B. (2012). Cannabis-related impairment and social anxiety: The roles of gender and cannabis use motives. Addictive behaviors, 37(11), 1294-1297.

Buckner, J. D., Heimberg, R. G., Ecker, A. H., \& Vinci, C. (2013). A biopsychosocial model of social anxiety and substance use. Depression and anxiety, 30(3), 276-284.

Burston, J. J., Wiley, J. L., Craig, A. A., Selley, D. E., \& Sim-Selley, L. J. (2010). Regional enhancement of cannabinoid CB1 receptor desensitization in female adolescent rats following repeated $\Delta 9$-tetrahydrocannabinol exposure. British journal of pharmacology, 161(1), 103-112.

Casey, B. J., Getz, S., \& Galvan, A. (2008). The adolescent brain. Developmental review, 28(1), 62-77.

Castillo, P. E., Younts, T. J., Chávez, A. E., \& Hashimotodani, Y. (2012). Endocannabinoid signaling and synaptic function. Neuron, 76(1), 70-81.

Castro-Schilo, L., \& Grimm, K. J. (2018). Using residualized change versus difference scores for longitudinal research. Journal of Social and Personal Relationships, 35(1), 32-58.

Chakravarthy, B., Shah, S., \& Lotfipour, S. (2013). Adolescent drug abuseAwareness \& prevention. The Indian journal of medical research, 137(6), 1021.

Chen, K., \& Kandel, D. B. (1995). The natural history of drug use from adolescence to the mid-thirties in a general population sample. American journal of public health, 85(1), 41-47.

Clark DB, Smith MG, Neighbors BD, Skerlec LM, and Randall J: Anxiety disorder in adolescence: characteristics, prevalence, and comorbidities. Clin Psychol Rev 1994; 14: pp. 113-137. 
Coles, M. E., \& Coleman, S. L. (2010). Barriers to treatment seeking for anxiety disorders: initial data on the role of mental health literacy. Depression and anxiety, 27(1), 63-71.

Compton, W. M., Han, B., Hughes, A., Jones, C. M., \& Blanco, C. (2017). Use of Marijuana for Medical Purposes Among Adults in the United States. JAMA, 317(2), 209-211.

Conway, K. P., Swendsen, J., Husky, M. M., He, J.-P., \& Merikangas, K. R. (2016). Association of lifetime mental disorders and subsequent alcohol and illicit drug use: results from the National Comorbidity Survey-Adolescent Supplement. Journal of the American Academy of Child \& Adolescent Psychiatry, 55(4), 280-288.

Crean, R. D., Crane, N. A., \& Mason, B. J. (2011). An evidence based review of acute and long-term effects of cannabis use on executive cognitive functions. Journal of addiction medicine, 5(1), 1.

Crippa, J. A., Zuardi, A. W., Martín-Santos, R., Bhattacharyya, S., Atakan, Z., McGuire, P., \& Fusar-Poli, P. (2009). Cannabis and anxiety: a critical review of the evidence. Human Psychopharmacology: Clinical and Experimental, 24(7), 515-523.

Dalton, V. S., \& Zavitsanou, K. (2010). Cannabinoid effects on CB1 receptor density in the adolescent brain: an autoradiographic study using the synthetic cannabinoid HU210. Synapse, 64(11), 845-854.

De Bellis, M. D., Wang, L., Bergman, S. R., Yaxley, R. H., Hooper, S. R., \& Huettel, S. A. (2013). Neural mechanisms of risky decision-making and reward response in adolescent onset cannabis use disorder. Drug and Alcohol Dependence, 133(1), 134145 .

Dodge, K. A., Malone, P. S., Lansford, J. E., Miller, S., Pettit, G. S., \& Bates, J. E. (2009). A dynamic cascade model of the development of substance-use onset. Monographs of the Society for Research in Child Development, 74(3), vii-119.

Duperrouzel, J., Hawes, S. W., Lopez-Quintero, C., Pacheco-Colón, I., Comer, J., \& Gonzalez, R. (2018). The association between adolescent cannabis use and anxiety: a parallel process analysis. Addictive behaviors, 78, 107-113.

Duperrouzel, J. C., Hawes, S. W., Lopez-Quintero, C., Pacheco-Colón, I., Coxe, S., Hayes, T., \& Gonzalez, R. (2019). Adolescent cannabis use and its associations with decision-making and episodic memory: Preliminary results from a longitudinal study. Neuropsychology. 
Eldreth, D. A., Matochik, J. A., Cadet, J. L., \& Bolla, K. I. (2004). Abnormal brain activity in prefrontal brain regions in abstinent marijuana users. NeuroImage, 23(3), 914-920.

Ellgren, M., Artmann, A., Tkalych, O., Gupta, A., Hansen, H. S., Hansen, S. H., ... \& Hurd, Y. L. (2008). Dynamic changes of the endogenous cannabinoid and opioid mesocorticolimbic systems during adolescence: THC effects. European Neuropsychopharmacology, 18(11), 826-834.

Ellickson, P. L., Martino, S. C., \& Collins, R. L. (2004). Marijuana use from adolescence to young adulthood: multiple developmental trajectories and their associated outcomes. Health Psychology, 23(3), 299.

Eysenck, M. W., Derakshan, N., Santos, R., \& Calvo, M. G. (2007). Anxiety and cognitive performance: attentional control theory. Emotion, 7(2), 336.

Feng, X., Shaw, D. S., \& Silk, J. S. (2008). Developmental trajectories of anxiety symptoms among boys across early and middle childhood. Journal of abnormal psychology, 117(1), 32.

Fergusson, D. M., \& Boden, J. M. (2008). Cannabis use and later life outcomes. Addiction, 103(6), 969-976.

Fergusson, D. M., \& Woodward, L. J. (2002). Mental health, educational, and social role outcomes of adolescents with depression. Archives of general psychiatry, 59(3), 225-231.

Flory, K., Lynam, D., Milich, R., Leukefeld, C., \& Clayton, R. (2004). Early adolescent through young adult alcohol and marijuana use trajectories: Early predictors, young adult outcomes, and predictive utility. Development and psychopathology, 16(1), 193-213.

Galbraith, S., Bowden, J., \& Mander, A. (2017). Accelerated longitudinal designs: An overview of modelling, power, costs and handling missing data. Statistical Methods in Medical Research, 26(1), 374-398.

Gonzalez, R., Schuster, R. M., Mermelstein, R. J., Vassileva, J., Martin, E. M., \& Diviak, K. R. (2012). Performance of young adult cannabis users on neurocognitive measures of impulsive behavior and their relationship to symptoms of cannabis use disorders. Journal of clinical and experimental neuropsychology, 34(9), 962-976.

Hale III, W. W., Raaijmakers, Q., Muris, P., \& Meeus, W. (2008). Developmental trajectories of adolescent anxiety disorder symptoms: A 5-year prospective community study. Journal of the American Academy of Child \& Adolescent Psychiatry, 47(5), 556-564. 
Hall, W., \& Degenhardt, L. (2000). Cannabis Use and Psychosis: A Review of Clinical and Epidemiological Evidence. Australian \& New Zealand Journal of Psychiatry, 34(1), 26-34.

Hampson, A. J., Grimaldi, M., Lolic, M., Wink, D., Rosenthal, R., \& Axelrod, J. (2000). Neuroprotective Antioxidants from Marijuanaa. Annals of the New York Academy of Sciences, 899(1), 274-282.

Hayatbakhsh, M. R., Najman, J. M., Jamrozik, K., Mamun, A. A., Alati, R., \& Bor, W. (2007). Cannabis and anxiety and depression in young adults: a large prospective study. Journal of the American Academy of Child \& Adolescent Psychiatry, 46(3), 408-417.

Heng, L., Beverley, J. A., Steiner, H., \& Tseng, K. Y. (2011). Differential developmental trajectories for CB1 cannabinoid receptor expression in limbic/associative and sensorimotor cortical areas. Synapse, 65(4), 278-286.

Hirvonen, J., Goodwin, R. S., Li, C.-T., Terry, G. E., Zoghbi, S. S., Morse, C., Innis, R. B. (2012). Reversible and regionally selective downregulation of brain cannabinoid CB1 receptors in chronic daily cannabis smokers. Molecular Psychiatry, 17(6), 642-649.

Howlett, A. C., Breivogel, C. S., Childers, S. R., Deadwyler, S. A., Hampson, R. E., \& Porrino, L. J. (2004). Cannabinoid physiology and pharmacology: 30 years of progress. Neuropharmacology, 47, 345-358.

Iversen, L. (2003). Cannabis and the brain. Brain: A Journal of Neurology, 126(Pt 6), $1252-1270$.

Johnston, L. D., Miech, R. A., O'Malley, P. M., Bachman, J. G., Schulenberg, J. E., \& Patrick, M. E. (2018). Monitoring the Future national survey results on drug use, 1975-2017: Overview, key findings on adolescent drug use.

Katona, I., Rancz, E. A., Acsády, L., Ledent, C., Mackie, K., Hájos, N., \& Freund, T. F. (2001). Distribution of CB1 cannabinoid receptors in the amygdala and their role in the control of GABAergic transmission. Journal of Neuroscience, 21(23), 95069518.

Kedzior, K. K., \& Laeber, L. T. (2014). A positive association between anxiety disorders and cannabis use or cannabis use disorders in the general population-a meta-analysis of 31 studies. BMC psychiatry, 14(1), 136.

Kessler, R. C., Ruscio, A. M., Shear, K., \& Wittchen, H. U. (2009). Epidemiology of anxiety disorders. In Behavioral neurobiology of anxiety and its treatment (pp. 2135). Springer, Berlin, Heidelberg. 
Khantzian, E. J. (1997). The self-medication hypothesis of substance use disorders: A reconsideration and recent applications. Harvard review of psychiatry, 4(5), 231-244.

King, S. M., Iacono, W. G., \& McGue, M. (2004). Childhood externalizing and internalizing psychopathology in the prediction of early substance use. Addiction, 99(12), 1548-1559.

Koob, G., \& Kreek, M. J. (2007). Stress, dysregulation of drug reward pathways, and the transition to drug dependence. American Journal of Psychiatry, 164(8), 11491159.

Kotov, R., Gamez, W., Schmidt, F., \& Watson, D. (2010). Linking "big” personality traits to anxiety, depressive, and substance use disorders: a metaanalysis. Psychological bulletin, 136(5), 768.

Langel, K., Engblom, C., Pehrsson, A., Gunnar, T., Ariniemi, K., \& Lillsunde, P. (2008). Drug testing in oral fluid - evaluation of sample collection devices. Journal of Analytical Toxicology, 32(6), 393-401.

Lantela, D. E., Kamal, B. S., \& Kamal, F. (2018). Cannabis and the Anxiety of Fragmentation-A Systems Approach for Finding an Anxiolytic Cannabis Chemotype. Frontiers in neuroscience, 12, 730.

Levin, I. P., \& Hart, S. S. (2003). Risk preferences in young children: Early evidence of individual differences in reaction to potential gains and losses. Journal of Behavioral Decision Making, 16(5), 397-413.

Levin, I. P., Hart, S. S., Weller, J. A., \& Harshman, L. A. (2007). Stability of choices in a risky decision-making task: A 3-year longitudinal study with children and adults. Journal of behavioral decision making, 20(3), 241-252.

Liu, W. J., Yin, D. Z., Cheng, W. H., Fan, M. X., You, M. N., Men, W. W., ... \& Zhang, F. (2015). Abnormal functional connectivity of the amygdala-based network in resting-state FMRI in adolescents with generalized anxiety disorder. Medical science monitor: international medical journal of experimental and clinical research, 21, 459.

Lorian, C. N., \& Grisham, J. R. (2011). Clinical implications of risk aversion: an online study of risk-avoidance and treatment utilization in pathological anxiety. Journal of Anxiety Disorders, 25(6), 840-848.

Lubman, D. I., Cheetham, A., \& Yücel, M. (2015). Cannabis and adolescent brain development. Pharmacology \& Therapeutics, 148, 1-16. 
Lyons, M. J., Bar, J. L., Panizzon, M. S., Toomey, R., Eisen, S., Xian, H., \& Tsuang, M. T. (2004). Neuropsychological consequences of regular marijuana use: a twin study. Psychological Medicine, 34(7), 1239-1250.

Lynskey, M., \& Hall, W. (2000). The effects of adolescent cannabis use on educational attainment: a review. Addiction, 95(11), 1621-1630.

Maldonado, R., Valverde, O., \& Berrendero, F. (2006). Involvement of the endocannabinoid system in drug addiction. Trends in neurosciences, 29(4), 225-232.

Maner, J. K., \& Schmidt, N. B. (2006). The role of risk avoidance in anxiety. Behavior Therapy, 37(2), 181-189.

Martin, M., Ledent, C., Parmentier, M., Maldonado, R., \& Valverde, O. (2002). Involvement of CB1 cannabinoid receptors in emotional behaviour.

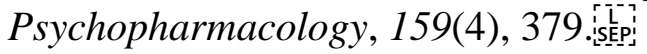

Mechoulam, R., \& Parker, L. A. (2013). The endocannabinoid system and the brain. Annual review of psychology, 64, 21-47.

Meier, M. H., Caspi, A., Ambler, A., Harrington, H., Houts, R., Keefe, R. S., ... \& Moffitt, T. E. (2012). Persistent cannabis users show neuropsychological decline from childhood to midlife. Proceedings of the National Academy of Sciences, 109(40), E2657-E2664.

Merikangas, K. R., \& Avenevoli, S. (2000). Implications of genetic epidemiology for the prevention of substance use disorders. Addictive behaviors, 25(6), 807-820.

Merikangas, K. R., He, J., Burstein, M., Swanson, S. A., Avenevoli, S., Cui, L., Swendsen, J. (2010). Lifetime prevalence of mental disorders in US adolescents: results from the National Comorbidity Survey Replication-Adolescent Supplement (NCS-A). Journal of the American Academy of Child \& Adolescent Psychiatry, 49(10), 980-989.

Miech RA, Johnston L, O’Malley PM, Bachman JG, Schulenberg J, Patrick ME. Trends in use of marijuana and attitudes toward marijuana among youth before and after decriminalization: The case of California 2007-2013. International Journal of Drug Policy. 2015;26(4):336-344.

Miech, R., Johnston, L., O'Malley, P., Bachman, J., Schulenberg, J., \& Patrick, M. (2019). Monitoring the Future national survey results on drug use, 1975-2018:

Volume I, Secondary school students. 
Moffitt, T. E., Harrington, H., Caspi, A., Kim-Cohen, J., Goldberg, D., Gregory, A. M., \& Poulton, R. (2007). Depression and generalized anxiety disorder: cumulative and sequential comorbidity in a birth cohort followed prospectively to age 32 years. Archives of general psychiatry, 64(6), 651-660.

Monk, C. S., Telzer, E. H., Mogg, K., Bradley, B. P., Mai, X., Louro, H. M., ... \& Pine, D. S. (2008). Amygdala and ventrolateral prefrontal cortex activation to masked angry faces in children and adolescents with generalized anxiety disorder. Archives of general psychiatry, 65(5), 568-576.

Muntoni, A. L., Pillolla, G., Melis, M., Perra, S., Gessa, G. L., \& Pistis, M. (2006). Cannabinoids modulate spontaneous neuronal activity and evoked inhibition of locus coeruleus noradrenergic neurons. European Journal of Neuroscience, 23(9), 23852394.

National Conference of State Legislatures (NCSL), State Medical Marijuana Laws (NCSL, 2019), http://www.ncsl.org/research/ health/state-medical-marijuanalaws.aspx

Nelemans, S. A., Hale, W. W., Raaijmakers, Q. A., Branje, S. J., van Lier, P. A., \& Meeus, W. H. (2016). Longitudinal associations between social anxiety symptoms and cannabis use throughout adolescence: the role of peer involvement. European child \& adolescent psychiatry, 25(5), 483-492.

Newman, M. G., Llera, S. J., Erickson, T. M., Przeworski, A., \& Castonguay, L. G. (2013). Worry and generalized anxiety disorder: a review and theoretical synthesis of evidence on nature, etiology, mechanisms, and treatment. Annual review of clinical psychology, 9, 275-297.

Ochsner, K. N., \& Gross, J. J. (2005). The cognitive control of emotion. Trends in Cognitive Sciences, 9(5), 242-249.44. Goldin, P. R., McRae, K., Ramel, W., \& Gross, J. J. (2008). The neural bases of emotion regulation: reappraisal and suppression of negative emotion. Biological Psychiatry, 63(6), 577-586.

O'shea, M., Singh, M. E., McGregor, I. S., \& Mallet, P. E. (2004). Chronic cannabinoid exposure produces lasting memory impairment and increased anxiety in adolescent but not adult rats.

Pacheco-Colón, I., Hawes, S. W., Duperrouzel, J. C., Lopez-Quintero, C., \& Gonzalez, R. (2019). Decision-Making as a Latent Construct and its Measurement Invariance in a Large Sample of Adolescent Cannabis Users. Journal of the International Neuropsychological Society, 1-7. 
Patton, G. C., Coffey, C., Carlin, J. B., Degenhardt, L., Lynskey, M., \& Hall, W. (2002). Cannabis use and mental health in young people: cohort

study. Bmj, 325(7374), 1195-1198.

Rey, A. A., Purrio, M., Viveros, M.-P., \& Lutz, B. (2012). Biphasic effects of cannabinoids in anxiety responses: $\mathrm{CB} 1$ and $\mathrm{GABAB}$ receptors in the balance of GABAergic and glutamatergic neurotransmission. Neuropsychopharmacology, 37(12), 2624-2634.

Rippeth, J. D., Heaton, R. K., Carey, C. L., Marcotte, T. D., Moore, D. J., Gonzalez, R., ... \& HNRC group. (2004). Methamphetamine dependence increases risk of neuropsychological impairment in HIV infected persons. Journal of the International Neuropsychological Society, 10(1), 1-14.

Ross, J. M., Coxe, S., Schuster, R. M., Rojas, A., \& Gonzalez, R. (2015). The moderating effects of cannabis use and decision making on the relationship between conduct disorder and risky sexual behavior. Journal of clinical and experimental neuropsychology, 37(3), 303-315.

Rubino, T., Sala, M., Viganò, D., Braida, D., Castiglioni, C., Limonta, V., Parolaro, D. (2007). Cellular Mechanisms Underlying the Anxiolytic Effect of Low Doses of Peripheral $\Delta 9$ - Tetrahydrocannabinol in Rats. Neuropsychopharmacology, 32(9), 2036-2045.

Schneider, M. (2008). Puberty as a highly vulnerable developmental period for the consequences of cannabis exposure. Addiction Biology, 13(2), 253-263.

Schulenberg, J. E., Merline, A. C., Johnston, L. D., O’Malley, P. M., Bachman, J. G., \& Laetz, V. B. (2005). Trajectories of Marijuana use during the Transition to Adulthood: The Big Picture Based on National Panel Data. Journal of Drug Issues, 35(2), 255-280.

Shaffer, D., Fisher, P., Lucas, C. P., Dulcan, M. K., \& Schwab-Stone, M. E. (2000). NIMH Diagnostic Interview Schedule for Children Version IV (NIMH DISC-IV): description, differences from previous versions, and reliability of some common diagnoses. Journal of the American Academy of Child \& Adolescent Psychiatry, 39(1), 28-38.

Sinha, R. (2008). Chronic stress, drug use, and vulnerability to addiction. Annals of the new York Academy of Sciences, 1141, 105.

Solomon, M. B., \& Herman, J. P. (2009). Sex differences in psychopathology: of gonads, adrenals and mental illness. Physiology \& behavior, 97(2), 250-258. 
Squeglia, L. M., Jacobus, J., \& Tapert, S. F. (2009). The Influence of Substance Use on Adolescent Brain Development. Clinical EEG and Neuroscience: Official Journal of the EEG and Clinical Neuroscience Society (ENCS), 40(1), 31-38.

Steinberg, L. (2005). Cognitive and affective development in adolescence. Trends in cognitive sciences, 9(2), 69-74.

Szabó, M. (2010). The short version of the Depression Anxiety Stress Scales (DASS21): Factor structure in a young adolescent sample. Journal of Adolescence, 33(1), 18.

Szuster RR, Pontius EB, Campos PE. Marijuana sensitivity and panic anxiety. Journal of Clinical Psychiatry. 1988;49:427-429.

Tunving, K. (1985). Psychiatric effects of cannabis use. Acta Psychiatrica Scandinavica, 72(3), 209-217.

Turner, S., Mota, N., Bolton, J., \& Sareen, J. (2018). Self-medication with alcohol or drugs for mood and anxiety disorders: A narrative review of the epidemiological literature. Depression and anxiety, 35(9), 851-860.

Van den Bergh, B. R. H., Van Calster, B., Puissant, S. P., \& Van Huffel, S. (2008). Self-reported symptoms of depressed mood, trait anxiety and aggressive behavior in post-pubertal adolescents: Associations with diurnal cortisol profiles. Hormones and behavior, 54(2), 253-257.

Van der Stelt, M., Veldhuis, W. B., Bär, P. R., Veldink, G. A., Vliegenthart, J. F. G., $\&$ Nicolay, K. (2001). Neuroprotection by $\Delta 9$-tetrahydrocannabinol, the main active compound in marijuana, against ouabain-induced in vivo excitotoxicity. Journal of Neuroscience, 21(17), 6475-6479.

Van Laar, M., Van Dorsselaer, S., Monshouwer, K., \& De Graaf, R. (2007). Does cannabis use predict the first incidence of mood and anxiety disorders in the adult population?. Addiction, 102(8), 1251-1260.

Viveros, M. P., Marco, E. M., \& File, S. E. (2005). Endocannabinoid system and stress and anxiety responses. Pharmacology Biochemistry and Behavior, 81(2), 331342.

Volkow, N. D., Baler, R. D., Compton, W. M., \& Weiss, S. R. (2014). Adverse health effects of marijuana use. New England Journal of Medicine, 370(23), 2219-2227.

Wadsworth, E., \& Hammond, D. (2018). Differences in patterns of cannabis use among youth: Prevalence, perceptions of harm and driving under the influence in the USA where non-medical cannabis markets have been established, proposed and prohibited. Drug and alcohol review, 37(7), 903-911. 
Walkup, J. T., Albano, A. M., Piacentini, J., Birmaher, B., Compton, S. N., Sherrill, J. T., ... \& Iyengar, S. (2008). Cognitive behavioral therapy, sertraline, or a combination in childhood anxiety. New England Journal of Medicine, 359(26), 2753-2766.

Wells, A., \& Carter, K. (1999). Preliminary tests of a cognitive model of generalized anxiety disorder. Behaviour Research and Therapy, 37(6), 585-594.

Wittchen, H. U., Fröhlich, C., Behrendt, S., Günther, A., Rehm, J., Zimmermann, P., ... \& Perkonigg, A. (2007). Cannabis use and cannabis use disorders and their relationship to mental disorders: a 10-year prospective-longitudinal community study in adolescents. Drug and alcohol dependence, 88, S60-S70.

Woodward, L. J., \& Fergusson, D. M. (2001). Life Course Outcomes of Young People With Anxiety Disorders in Adolescence. Journal of the American Academy of Child \& Adolescent Psychiatry, 40(9), 1086-1093.

Zhao, X. H., Wang, P. J., Li, C. B., Hu, Z. H., Xi, Q., Wu, W. Y., \& Tang, X. W. (2007). Altered default mode network activity in patient with anxiety disorders: an fMRI study. European journal of radiology, 63(3), 373-378.

Zvolensky, M. J., Lewinsohn, P., Bernstein, A., Schmidt, N. B., Buckner, J. D., Seeley, J., \& Bonn-Miller, M. O. (2008). Prospective associations between cannabis use, abuse, and dependence and panic attacks and disorder. Journal of psychiatric research, 42(12), 1017-1023. 
APPENDICES 
Table 1: Descriptive Statistics for Study Sample at Baseline

\begin{tabular}{|c|c|c|c|}
\hline & $\begin{array}{l}\text { Minimal Trajectory } \\
(n=262)\end{array}$ & $\begin{array}{l}\text { Escalating Trajectory } \\
(\mathrm{n}=\mathbf{7 8})\end{array}$ & $\begin{array}{c}\text { Chronic Trajectory } \\
(\mathrm{n}=61)\end{array}$ \\
\hline Age & $15.64(.70)_{\mathrm{A}}$ & $15.88(.63)$ в & $15.65(.65)_{\mathrm{A}}$ \\
\hline \multicolumn{4}{|l|}{ Years of Education } \\
\hline Years of Education (Mother) & $14.34(2.42)$ & $14.05(2.75)$ & $14.00(2.49)$ \\
\hline Years of Education (Father) & $13.98(2.86)$ & $13.36(2.92)$ & $13.36(3.00)$ \\
\hline WRAT-4 Reading Standard Score & $108.52(13.9)$ & $108.68(14.73)$ & $106.93(14.53)$ \\
\hline \multicolumn{4}{|l|}{ Ethnicity/race $(\%)$} \\
\hline Hispanic/Latino & $91 \%$ & $82 \%$ & $96 \%$ \\
\hline Caucasian & $4 \%$ & $7 \%$ & $2 \%$ \\
\hline African-American & $4 \%$ & $10 \%$ & $2 \%$ \\
\hline Other & $1 \%$ & $1 \%$ & $0 \%$ \\
\hline Female $(\%)$ & $57 \% \mathrm{~A}$ & $23 \%$ в & $28 \%$ в \\
\hline \multicolumn{4}{|l|}{ Substance Use } \\
\hline Amount of Lifetime Nicotine (cigarettes; MD, IQR) & $0(0, .25)_{\mathrm{A}}$ & $0(0,5.75)_{\mathrm{A}}$ & $2(0,29)_{\mathrm{B}}$ \\
\hline Frequency of Lifetime Nicotine (cigarettes; MD, IQR) & $0(0,1)_{\mathrm{A}}$ & $0(0,6)_{\mathrm{A}}$ & $2(0,24.5)$ в \\
\hline Amount of Lifetime Alcohol (1 serving; MD, IQR) & $5(1,39)_{\mathrm{A}}$ & $18(3.38,65.75) \mathrm{AB}$ & $20(7.75,110.5)_{\text {в }}$ \\
\hline Frequency of Lifetime Alcohol (1 serving; MD, IQR) & $3(1,16)_{\mathrm{A}}$ & $7.5(2,21.75)_{\mathrm{AB}}$ & $10(2,40)$ в \\
\hline Amount of Lifetime Cannabis (g; MD, IQR) & $1.88(0,15.98)_{\mathrm{A}}$ & $61.34(17.28,174.63)_{\text {в }}$ & $172.95(76,376)_{\mathrm{C}}$ \\
\hline Frequency of Lifetime Cannabis (days, MD, IQR) & $6(0,32)_{\mathrm{A}}$ & $82.5(36.5,256)$ в & $309(180,597) \mathrm{C}$ \\
\hline Ever Used Cannabis $(\%)$ & $68 \% \mathrm{~A}$ & $100 \%$ в & $97 \%$ в \\
\hline Used Cannabis in Past Month (\%) & $35 \% \mathrm{~A}$ & $85 \%$ в & $92 \% \mathrm{c}$ \\
\hline Used Cannabis in Past Six Months (\%) & $60 \% \mathrm{~A}$ & $96 \%$ в & $95 \% \mathrm{C}$ \\
\hline Used Cannabis in Past Year (\%) & $65 \% \mathrm{~A}$ & $100 \%$ в & $97 \% \mathrm{c}$ \\
\hline $\begin{array}{l}\text { Ever Used Alcohol }(\%) \\
\text { E }\end{array}$ & $77 \% \mathrm{~A}$ & $94 \%$ в & $92 \%$ в \\
\hline $\begin{array}{l}\text { Ever Used Alcohol (\%) } \\
\text { Ever Used Nicotine (\%) }\end{array}$ & $32 \% \mathrm{~A}$ & $49 \%$ в & $70 \% \mathrm{c}$ \\
\hline
\end{tabular}

Note: Abbreviations: WRAT-4, Wide Range Achievement Test; DASS-21, SD, standard deviation; Md, median; IQR, interquartile range. Superscript letters indicate significant mean differences between groups $(p<.05)$. 
Table 2: Participant Characteristics for Aim 1

\begin{tabular}{|c|c|c|c|}
\hline & $\begin{array}{l}\text { Minimal Trajectory } \\
(\mathrm{n}=\mathbf{2 6 2})\end{array}$ & $\begin{array}{c}\text { Escalating Trajectory } \\
\quad(n=78)\end{array}$ & $\begin{array}{l}\text { Chronic Trajectory } \\
\quad(n=61)\end{array}$ \\
\hline $\begin{array}{l}\text { T5 Lifetime Frequency of Cannabis Use } \\
\quad(g ; M D, I Q R)\end{array}$ & $15(0,70.5)_{\mathrm{A}}$ & $218(122.75,418)$ в & $529(414.5,804)_{\mathrm{C}}$ \\
\hline $\begin{array}{l}\text { T5 Lifetime Amount of Cannabis Use } \\
(g ; M D, I Q R)\end{array}$ & $4.9(0,32.83)_{\mathrm{A}}$ & $171.86(71.69,363.89)_{\mathrm{B}}$ & $479(249.92,752.50)_{\mathrm{C}}$ \\
\hline $\begin{array}{l}\text { T5 Lifetime Frequency of Alcohol Use } \\
\text { (1 serving; } M D, I Q R)\end{array}$ & $17(6,54.5)_{\mathrm{A}}$ & $35(14.25,108.25)$ в & $43(17,125.5)$ в \\
\hline $\begin{array}{l}\text { T5 Lifetime Amount of Alcohol Use } \\
\text { (1 serving; } M D, I Q R)\end{array}$ & $48.16(12.13,182.83)_{\mathrm{A}}$ & $157(38,395.2)_{\text {в }}$ & $156(56,558.5)_{\mathrm{C}}$ \\
\hline $\begin{array}{l}\text { T5 Lifetime Frequency of Nicotine } \\
\text { (cigarettes; } M D, I Q R)\end{array}$ & $4(0,20)_{\mathrm{A}}$ & $104.5(45,176.25)$ в & $186(151,258.5)_{\mathrm{C}}$ \\
\hline $\begin{array}{l}\text { T5 Lifetime Amount of Nicotine } \\
\text { (cigarettes; } M D, I Q R)\end{array}$ & $2(0,20)_{A}$ & $86.26(37.36,224.69)_{\mathrm{AB}}$ & $\begin{array}{l}222.75(115.5 \\
409.73)_{\text {в }}\end{array}$ \\
\hline \multicolumn{4}{|l|}{ Mental Health, Mean (SD) } \\
\hline DASS-21 Anxiety Z-Score at T1 & $-.30(.90)$ & $-.34(.86)$ & $-.31(.70)$ \\
\hline DASS-21 Anxiety Z-Score at T5 & $-.33(1.06)_{\mathrm{A}}$ & $.16(1.51)_{\mathrm{B}}$ & $.04(1.07) \mathrm{B}$ \\
\hline DASS-21 Depression Z-Score at T1 & $-.30(.90)$ & $.34(.86)$ & $-.31(.70)$ \\
\hline DASS-21 Depression Z-Score at T5 & $-.42(.90)$ & $-.17(.80)$ & $-.23(.96)$ \\
\hline DASS-21 Stress Z-Score at T1 & $-.44(.90)$ & $-.54(.80)$ & $-.48(.76)$ \\
\hline DASS-21 Stress Z-Score at T5 & $-.62(.84)$ & $-.42(1.13)$ & $-57(.92)$ \\
\hline
\end{tabular}

Note: Abbreviations: Depression, Anxiety, and Stress Scale, Short Version (DASS-21); Md, median; IQR, interquartile range. Superscript letters indicate significant mean differences between groups $(p<.05)$. 
Table 3: Participant Characteristics for Aim 2

\begin{tabular}{|c|c|c|c|}
\hline & $\begin{array}{l}\text { Minimal Trajectory } \\
(\mathrm{n}=\mathbf{2 6 2})\end{array}$ & $\begin{array}{l}\text { Escalating Trajectory } \\
\qquad(\mathrm{n}=78)\end{array}$ & $\begin{array}{l}\text { Chronic Trajectory } \\
\quad(n=61)\end{array}$ \\
\hline T3 Lifetime Frequency of Cannabis Use $(g, M D, I Q R)$ & $15(0,70.5) \mathrm{A}$ & $218(122.75,418)_{\text {в }}$ & $529(414.5,804) \mathrm{C}$ \\
\hline T3 Lifetime Amount of Cannabis Use $(g, M D, I Q R)$ & $4.9(0,32.83)_{\mathrm{A}}$ & $171.86(71.69,363.89)_{\mathrm{B}}$ & $479(249.92,752.50)_{\mathrm{C}}$ \\
\hline T5 Lifetime Frequency of Cannabis Use $(g, M D, I Q R)$ & $23(0,108.5) \mathrm{A}$ & $451(280,608)_{\text {в }}$ & $818(644,1032)_{\mathrm{C}}$ \\
\hline T5 Lifetime Amount of Cannabis Use $(g, M D, I Q R)$ & $7.65(0,61.9)_{\mathrm{A}}$ & $353(192.83,605.9)_{\text {в }}$ & $806.41(474.57,1275.78) \mathrm{C}$ \\
\hline $\begin{array}{l}\text { T3 Lifetime Frequency of Alcohol Use } \\
\text { (1 serving; } M D, I Q R)\end{array}$ & $10(2,32)_{\mathrm{A}}$ & $19.5(6,52)_{\mathrm{A}}$ & $20(6.5,81)$ в \\
\hline $\begin{array}{l}\text { T3 Lifetime Amount of Alcohol Use } \\
\text { (1 serving; } M D, I Q R)\end{array}$ & $19(3,90.33) \mathrm{A}$ & $59.5(17,179.5) \mathrm{A}$ & $64(24,290.5)$ в \\
\hline $\begin{array}{l}\text { T5 Lifetime Frequency of Alcohol Use } \\
\text { (1 serving; } M D, I Q R)\end{array}$ & $17(6,54.5)_{\mathrm{A}}$ & $35(14.25,108.25)$ в & $43(17,125.5)$ в \\
\hline $\begin{array}{l}\text { T5 Lifetime Amount of Alcohol Use } \\
\text { (1 serving; } M D, I Q R)\end{array}$ & $48.16(12.13,182.83)_{\mathrm{A}}$ & $157(38,395.2)$ в & $156(56,558.5)_{\mathrm{C}}$ \\
\hline T3 Lifetime Frequency of Nicotine (cigarettes; MD, IQR) & $0(0,3.5)_{\mathrm{A}}$ & $1(0.13 .5)$ в & $15(1,56)_{C}$ \\
\hline T3 Lifetime Amount of Nicotine (cigarettes; MD, IQR) & $0(0,3) \mathrm{A}$ & $0(.59,33.5)_{\mathrm{AB}}$ & $22.75(.25,122)$ в \\
\hline T5 Lifetime Frequency of Nicotine (cigarettes; MD, IQR) & $4(0,20)_{\mathrm{A}}$ & $104.5(45,176.25)_{\text {в }}$ & $186(151,258.5)_{\mathrm{C}}$ \\
\hline T5 Lifetime Amount of Nicotine (cigarettes; MD, IQR) & $2(0,20)_{\mathrm{A}}$ & $86.26(37.36,224.69)_{\mathrm{AB}}$ & $222.75(115.5,409.73)$ в \\
\hline \multicolumn{4}{|l|}{ Mental Health, Mean (SD) } \\
\hline DASS-21 Anxiety Z-Score at T3 & $-.20(1.20)$ & $.14(1.37)$ & $.15(1.15)$ \\
\hline DASS-21 Depression Z-Score at T3 & $-.28(.99)$ & $-.37(.79)$ & $-.22(.98)$ \\
\hline DASS-21 Stress Z-Score at T3 & $-.51(.95)$ & $-.49(.87)$ & $-.50(.90)$ \\
\hline C-DISC GAD Symptoms at T3 & $.50(.92) \mathrm{A}$ & $.49(.93)$ в & $.59(1.10)_{\mathrm{AB}}$ \\
\hline C-DISC PD Symptoms at T3 & $2.81(2.31)$ & $2.38(2.32)$ & $1.88(1.81)$ \\
\hline C-DISC GAD Symptoms T5 & $.46(.91)$ & $.39(.92)$ & $.47(.82)$ \\
\hline C-DISC PD Symptoms at T5 & $2.18(2.12)$ & $2.17(2.07)$ & $1.85(1.78)$ \\
\hline
\end{tabular}

Note: Abbreviations: DASS-21, Depression, Anxiety, Stress, Scale, 21-item version; Computer Diagnostic Interview Schedule for Children, Fourth Edition (C-DISC); Md, median; IQR, interquartile range. Superscript letters indicate significant mean differences between groups $(p<.05)$. 
Table 4: Participant Characteristics for Aim 3

\begin{tabular}{|c|c|c|c|}
\hline & $\begin{array}{l}\text { Minimal Trajectory } \\
(n=262)\end{array}$ & $\begin{array}{c}\text { Escalating Trajectory } \\
(\mathrm{n}=78)\end{array}$ & $\begin{array}{c}\text { Chronic Trajectory } \\
(\mathrm{n}=61)\end{array}$ \\
\hline \multicolumn{4}{|l|}{ Substance Use, MD (IQR) } \\
\hline T5 Lifetime Frequency of Cannabis Use (grams) & $15(0,70.5) \mathrm{A}$ & $218(122.75,418)$ в & $529(414.5,804) \mathrm{C}$ \\
\hline T5 Lifetime Amount of Cannabis Use (grams) & $4.9(0,32.83)_{\mathrm{A}}$ & $171.86(71.69,363.89)_{\text {в }}$ & $479(249.92,752.50)_{\mathrm{C}}$ \\
\hline $\begin{array}{l}\text { T5 Lifetime Frequency of Alcohol Use } \\
\text { (days) }\end{array}$ & $17(6,54.5)_{\mathrm{A}}$ & $35(14.25,108.25)$ в & $43(17,125.5)$ в \\
\hline $\begin{array}{l}\text { T5 Lifetime Amount of Alcohol Use } \\
\text { (1 serving) }\end{array}$ & $48.16(12.13,182.83)_{\mathrm{A}}$ & $157(38,395.2)$ в & $156(56,558.5)_{\mathrm{C}}$ \\
\hline T5 Lifetime Frequency of Nicotine (days) & $4(0,20)_{\mathrm{A}}$ & $104.5(45,176.25)$ в & $186(151,258.5)_{\mathrm{C}}$ \\
\hline T5 Lifetime Amount of Nicotine (cigarettes) & $2(0,20)_{\mathrm{A}}$ & $86.26(37.36,224.69)_{\mathrm{AB}}$ & $\begin{array}{c}222.75(115.5 \\
409.73)_{\text {в }}\end{array}$ \\
\hline \multicolumn{4}{|l|}{ Mental Health, Mean (SD) } \\
\hline DASS-21 Anxiety Z-Score at T5 & $-.33(1.06)_{\mathrm{A}}$ & $16(1.51)$ в & $.04(1.07)$ в \\
\hline DASS-21 Depression Z-Score at T5 & $-.42(.90)$ & $-.17(.80)$ & $-.23(.96)$ \\
\hline DASS-21 Stress Z-Score at T5 & $-.62(.84)$ & $-.42(1.13)$ & $-57(.92)$ \\
\hline \multicolumn{4}{|l|}{ Decision Making Measures, Mean (SD) } \\
\hline IGT Net Total Reverse Scored & $-11.83(28.99)$ & $-6.68(27.96)$ & $-6.68(27.50)$ \\
\hline Cups Gain Total & $17.34(5.59)$ & $16.54(5.06)$ & $17.67(5.49)$ \\
\hline Cups Loss Total & $17.78(6.09)$ & $16.74(6.37)$ & $15.77(6.45)$ \\
\hline GDT Total Risky Trials & $5.24(4.66)$ & $5.68(4.98)$ & $6.23(5.01)$ \\
\hline
\end{tabular}

Note: Abbreviations: DASS-21, Depression, Anxiety, Stress, Scale, 21-item version; Iowa Gabling Test; GDT, Game of Dice Task; SD, standard deviation; Md, median; IQR, interquartile range. Neurocognitive performance was assessed at T5. Superscript letters indicate significant mean differences between groups $(p<.05)$. 
Table 5: Fit Information for Latent Class Growth Analyses of Cannabis Trajectories

\begin{tabular}{llll}
\hline & BIC & Entropy & BLRT \\
\cline { 2 - 4 } 2-Class & 18700.77 & .960 & $p=0.00$ \\
3-Class & 18465.28 & .922 & $p=0.00$ \\
4-Class & 18368.17 & .916 & $p=0.00$ \\
\hline
\end{tabular}

Note. Abbreviations: BIC=Sample size adjusted Bayesian Information Criterion. BLRT = Bootstrapped Likelihood Ratio. 
Table 6: Results of cannabis use trajectory membership predicting changes in anxiety symptomology across two-years

\section{Trajectory Class Membership Comparisons 1}

Minimal vs. Escalating CU Minimal vs. Chronic CU Chronic v. Escalating CU

Predictors

$\begin{array}{llll}\text { DASS-21 Change } & -.37(.18) p=.03^{*} & .11(.15) p=.46 & -.77(.34) p=.04 * \\ \text { PD Symptoms Change } & -.29(.17) p=.09 & .15(.13) p=.28 & -.14(.25), p=.58 \\ \text { GAD Symptoms Change } & -.17(.13) p=.19 & -.06(.12) p=.62 & .26(.17) p=.12\end{array}$

Note. Abbreviations: Cannabis Use (CU); Depression, Anxiety, and Stress Scale, Short Version (DASS-21); Panic Disorder (PD); Generalized Anxiety Disorder (GAD). Linear regression statistics by comparison class presented with standardized estimates,

standard error, and p-value. 1 First CU trajectory group listed for each comparison serves as the reference group. $* \mathrm{p}<.05$; $* * * \mathrm{p}<.001$ 
Table 7: Results of cannabis use trajectory membership predicting panic disorder and general anxiety disorder membership at 1year and two-year follow-up

\section{Trajectory Class Membership Comparisons1}

\begin{tabular}{lll} 
Chronic vs. Minimal CU & Chronic vs. Escalating CU & Minimal v. Escalating CU \\
$O R(95 \% \mathrm{CI})$ & $O R(95 \% \mathrm{CI})$ & $O R(95 \%$ \\
\hline
\end{tabular}

Predictors

$\begin{array}{llll}\text { Panic Disorder T3 } & 1.11(.11,11.2) & .80(.15,4.27) & .39(.10,1.43)^{*} \\ \text { Panic Disorder T5 } & \infty 2 & \infty 2 & .63(.16,2.51) \\ \text { Generalized Anxiety T3 } & \infty 2 & \infty 2 & 1.31(.31,5.57) \\ \text { Generalized Anxiety T5 } & \infty 2 & & .82(.17,2.40)\end{array}$

Note. Abbreviations: Cannabis Use (CU). ${ }_{1}$ Odds ratios derived from logistic regression estimates in Mplus. $\infty_{2}=$ not applicable: odds ratio could not be calculated because one of the cells contained zero: participants in the chronic trajectory did not have panic disorder at T5, or generalized anxiety disorder at T3 or T5. First CU trajectory group listed for each comparison serves as the reference group. $* \mathrm{p}<.05 ; * * \mathrm{p}<.001$ 
Table 8. Model parameter estimates of the interactive effects of anxiety and cannabis use trajectory membership with decision-making at two-year follow-up

\begin{tabular}{llll}
\hline Paths & $\begin{array}{l}\text { Standardized } \\
\text { Estimate }\end{array}$ & $\begin{array}{l}\text { Unstandardized } \\
\text { Estimate }(\boldsymbol{S E})\end{array}$ & p-value \\
\hline Decision Making Main Model & & & $p=.497$ \\
CU Group $\rightarrow$ Decision Making & -.04 & .013 & $p=035$ \\
Anxiety $\rightarrow$ Decision Making* & -30 & .025 & $p=.041$ \\
GroupXAnxiety $\rightarrow$ Decision Making* & .24 & .010 & $p<.001$ \\
Gender $\rightarrow$ Decision Making** & .19 & .025 & $P=.036$ \\
FSIQ $\rightarrow$ Decision Making* & -.12 & .11 & $p=.021$ \\
Alcohol $\rightarrow$ Decision Making* & .12 & .000 & $p=.922$ \\
Nicotine $\rightarrow$ Decision Making & -.01 & .022 &
\end{tabular}


Figure 1: Proposed Biopsychosocial Model of Anxiety and Cannabis Use

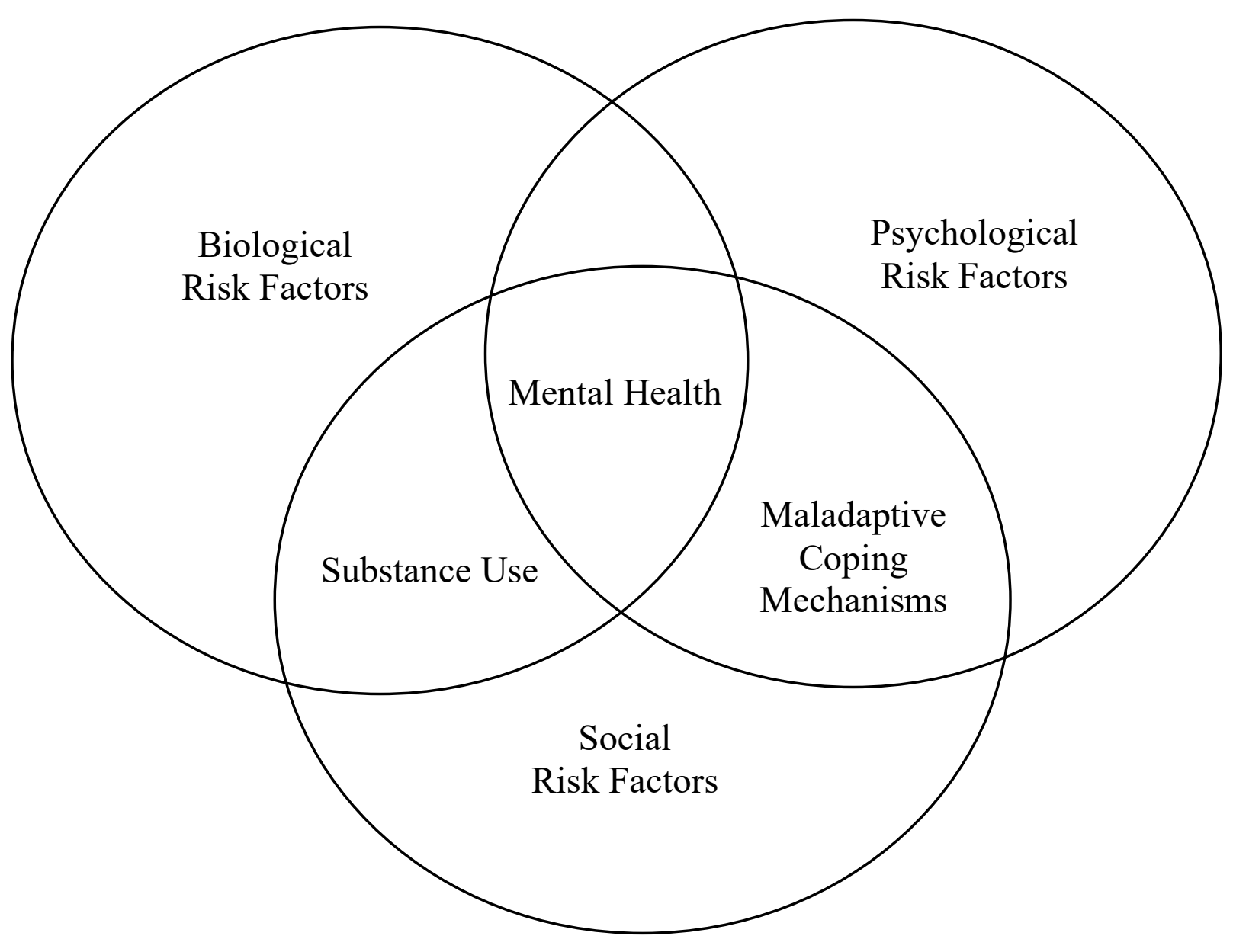


Figure 2: Reinforcement Model of Anxiety through Self-Medicating with Cannabis Use

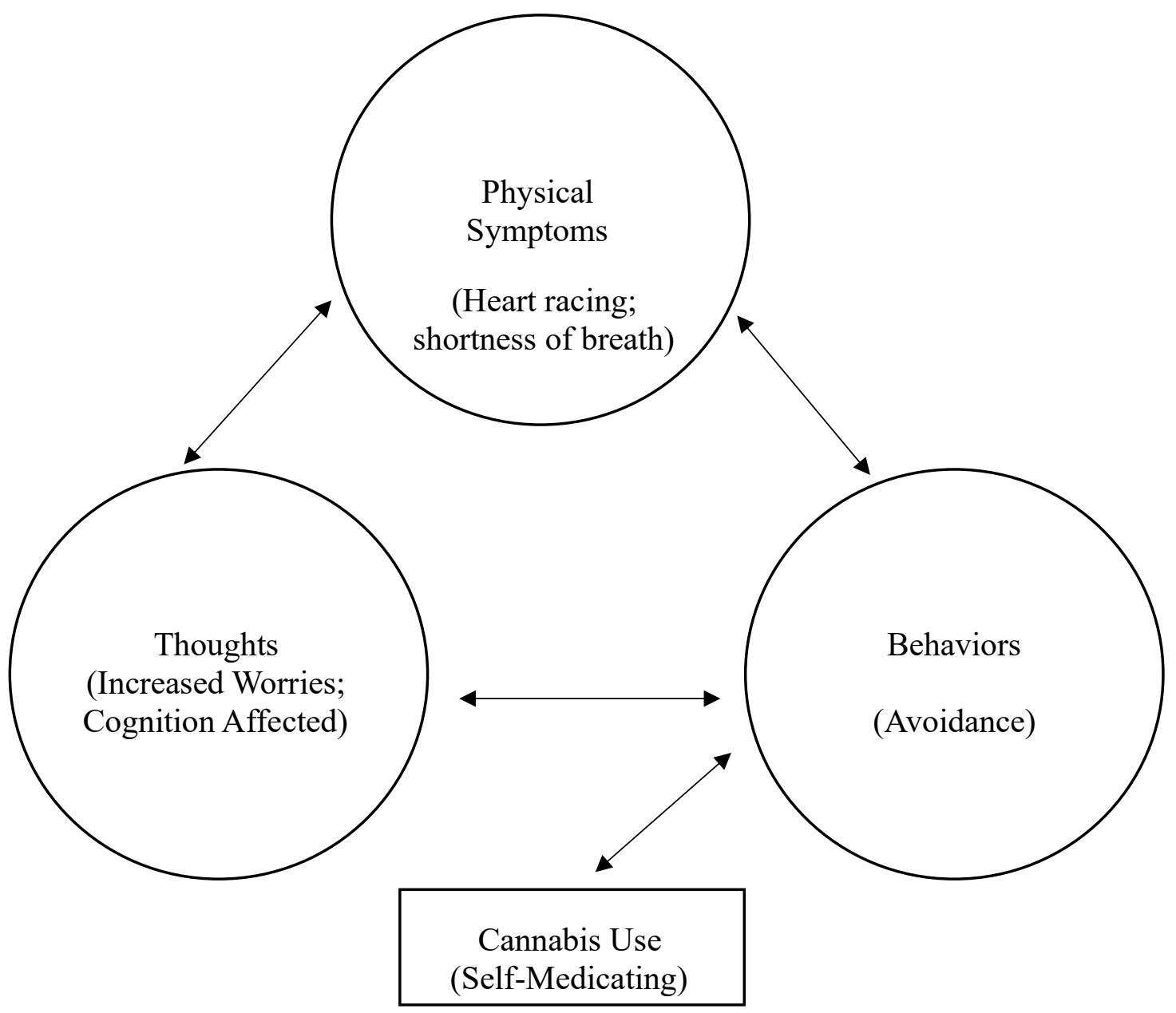


Figure 3: Assessment Timeline from Baseline to T5

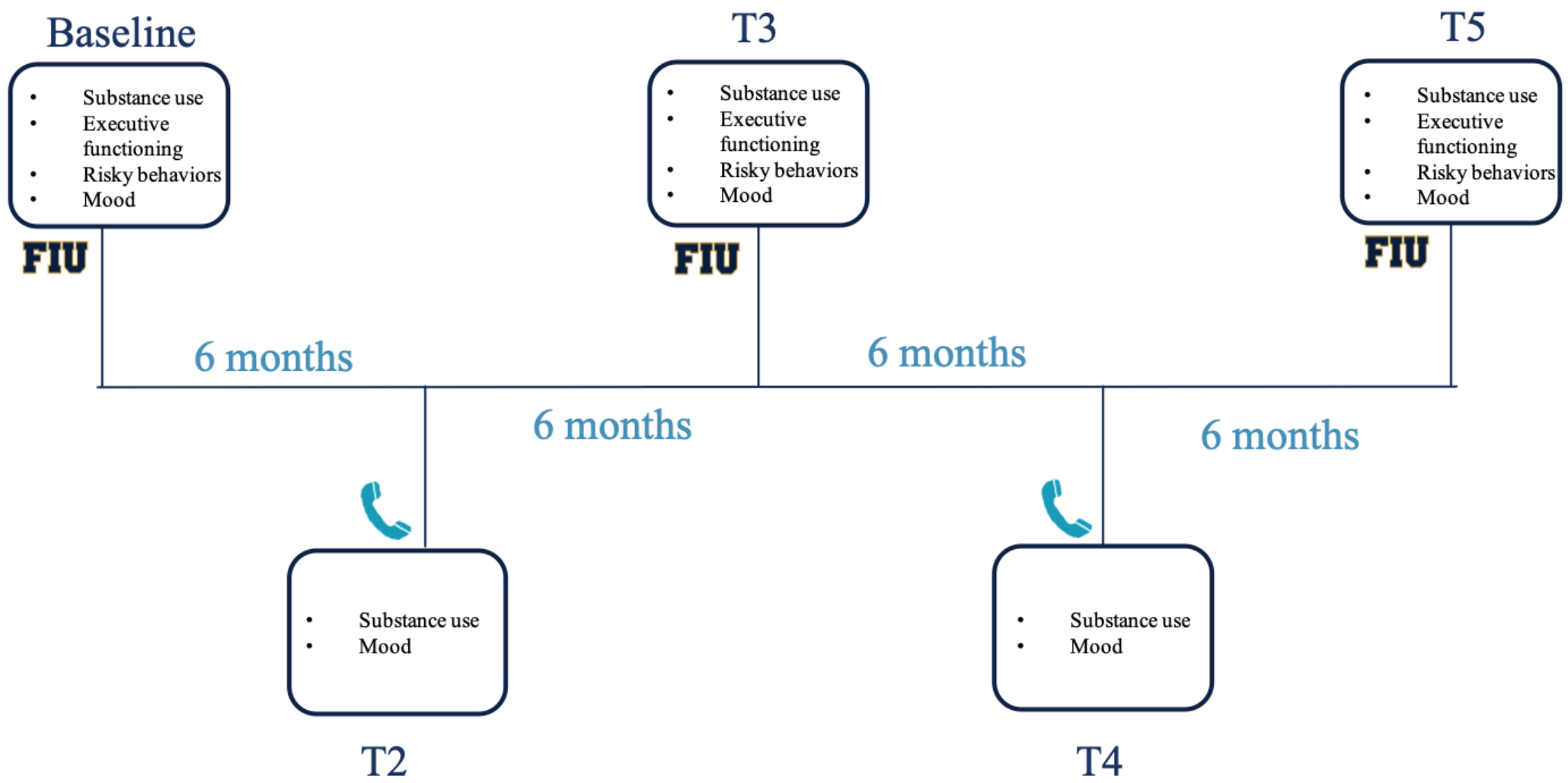


Figure 4: Trajectories of Adolescent Cannabis Use at Baseline - Three Classes

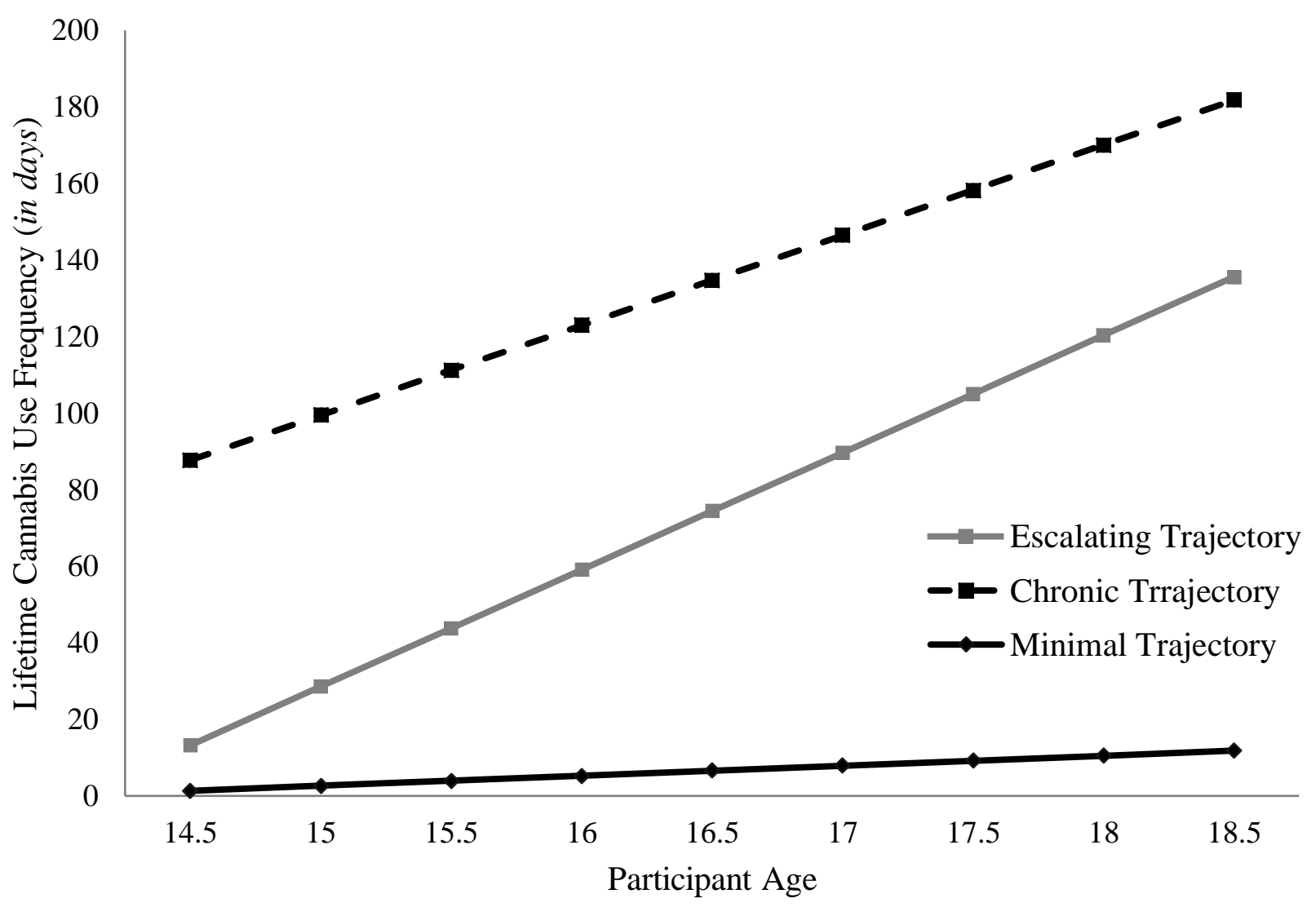


Figure 5: Trajectories of Adolescent Cannabis Use at Baseline - Four Classes

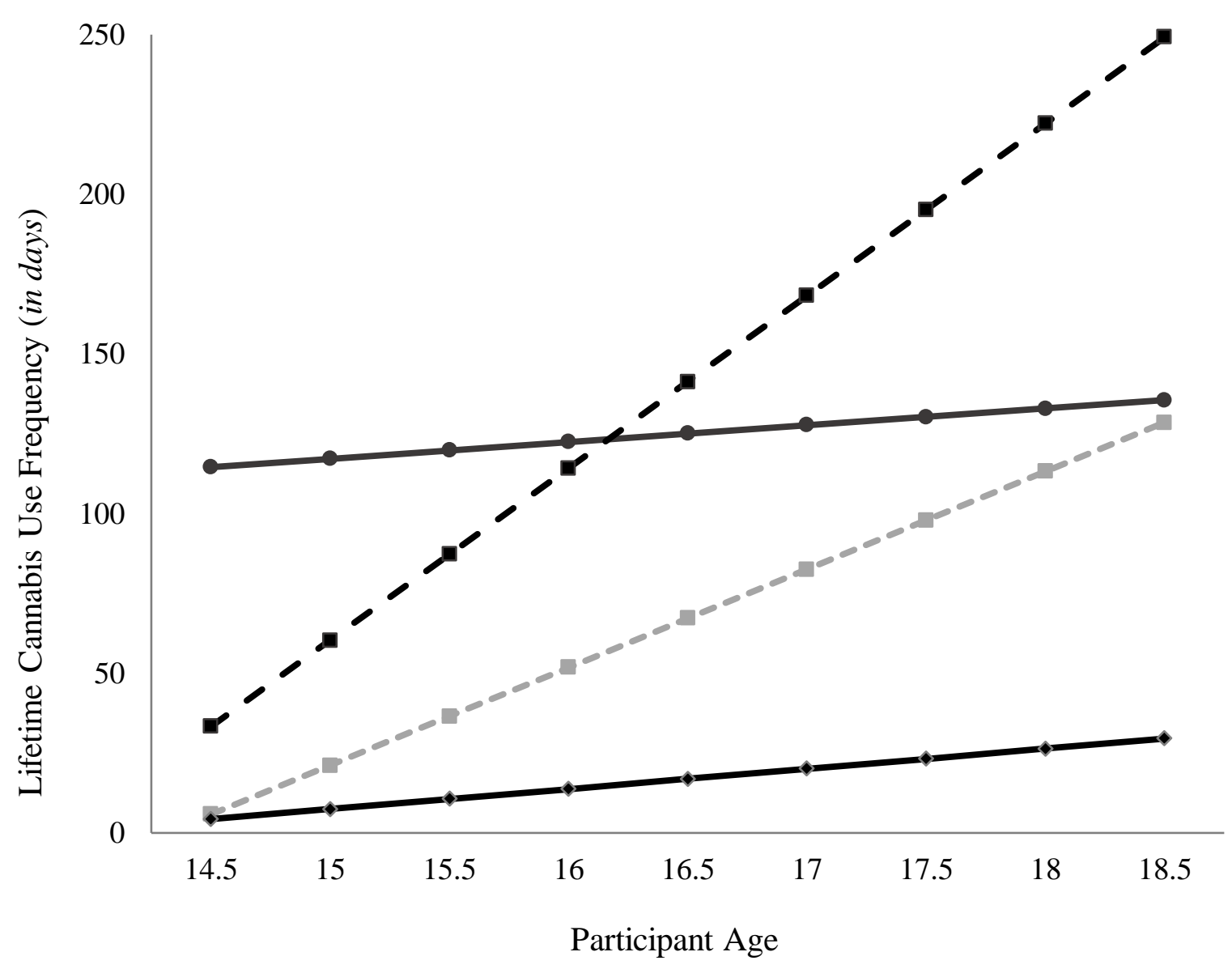


Figure 6: Changes in DASS-21 Anxiety Symptoms by Cannabis Use Trajectory from T1-T5

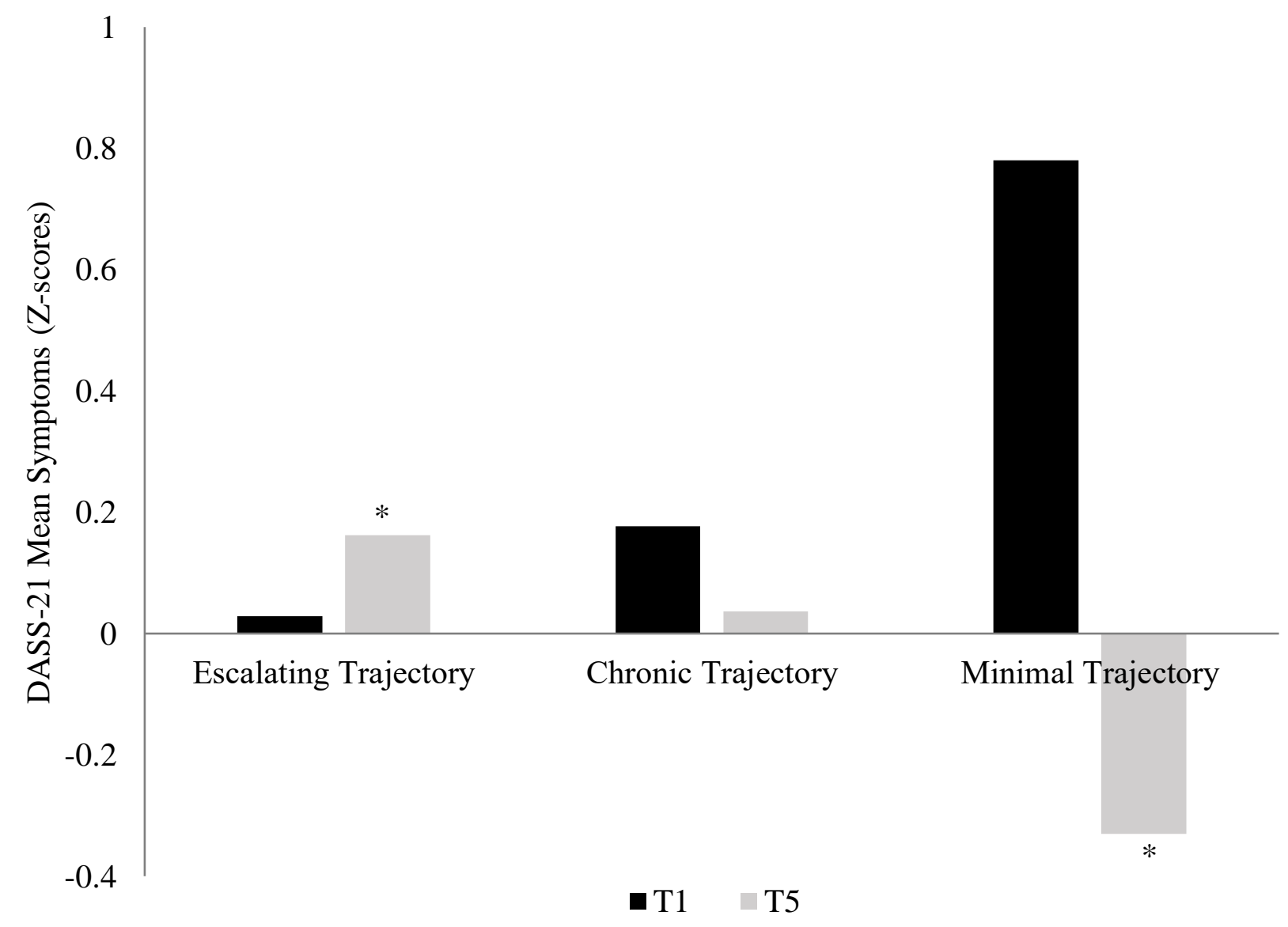


Figure 7: Changes in PD Symptoms by Cannabis Use Trajectory from T1-T5

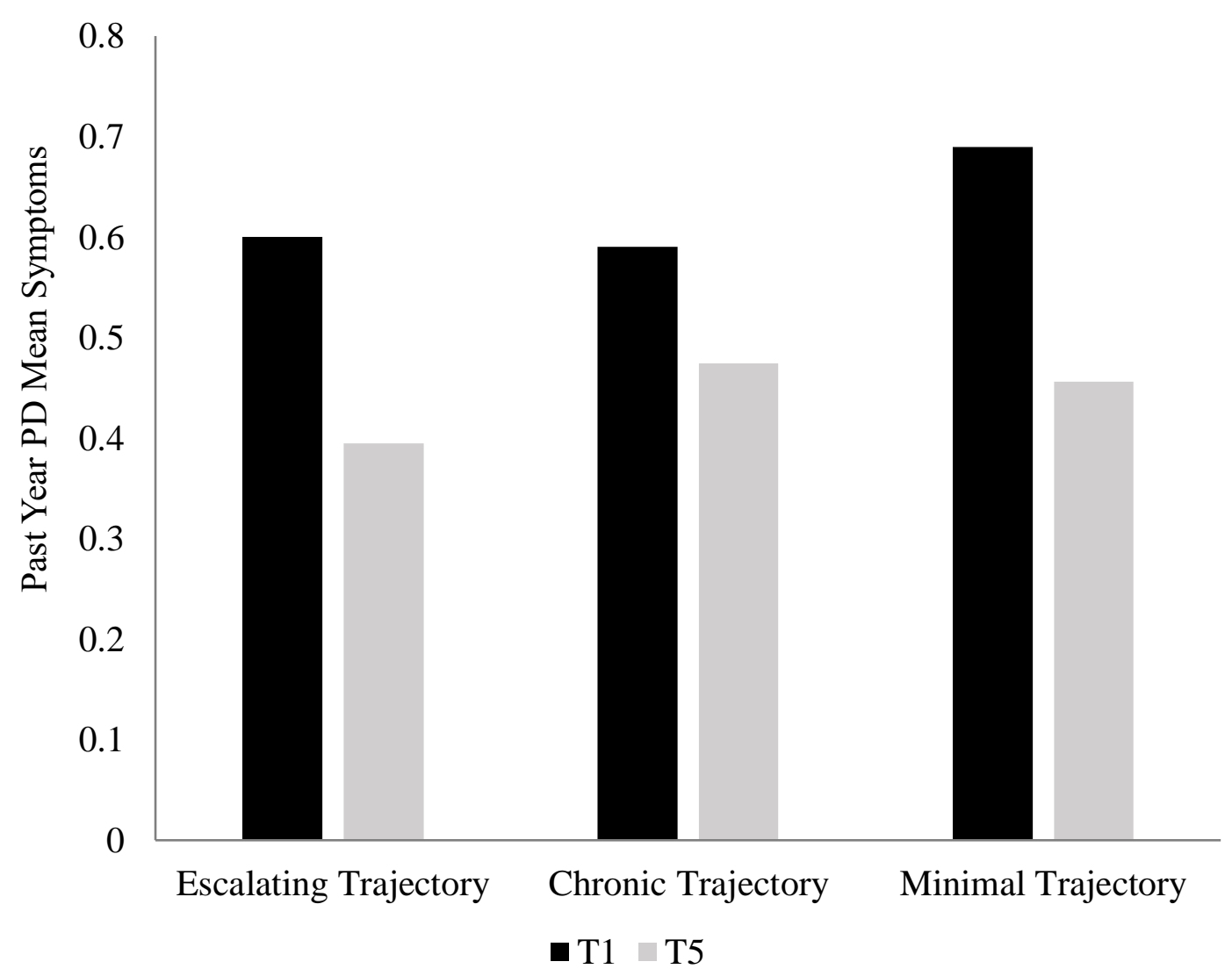


Figure 8: Changes in GAD Symptoms by Cannabis Use Trajectory from T1-T5

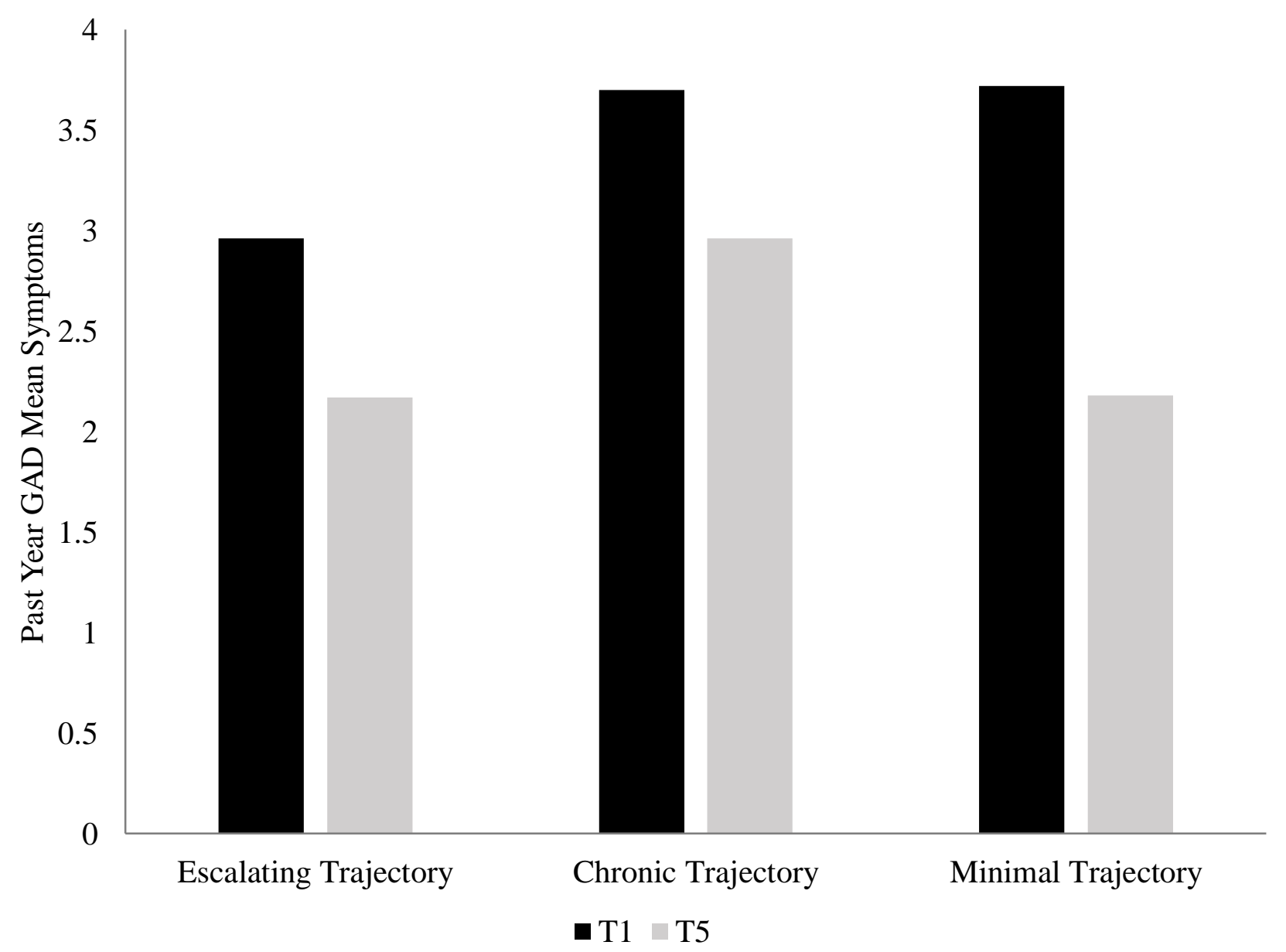


Figure 9: Mean Unstandardized Anxiety Residuals by Cannabis Use Trajectory

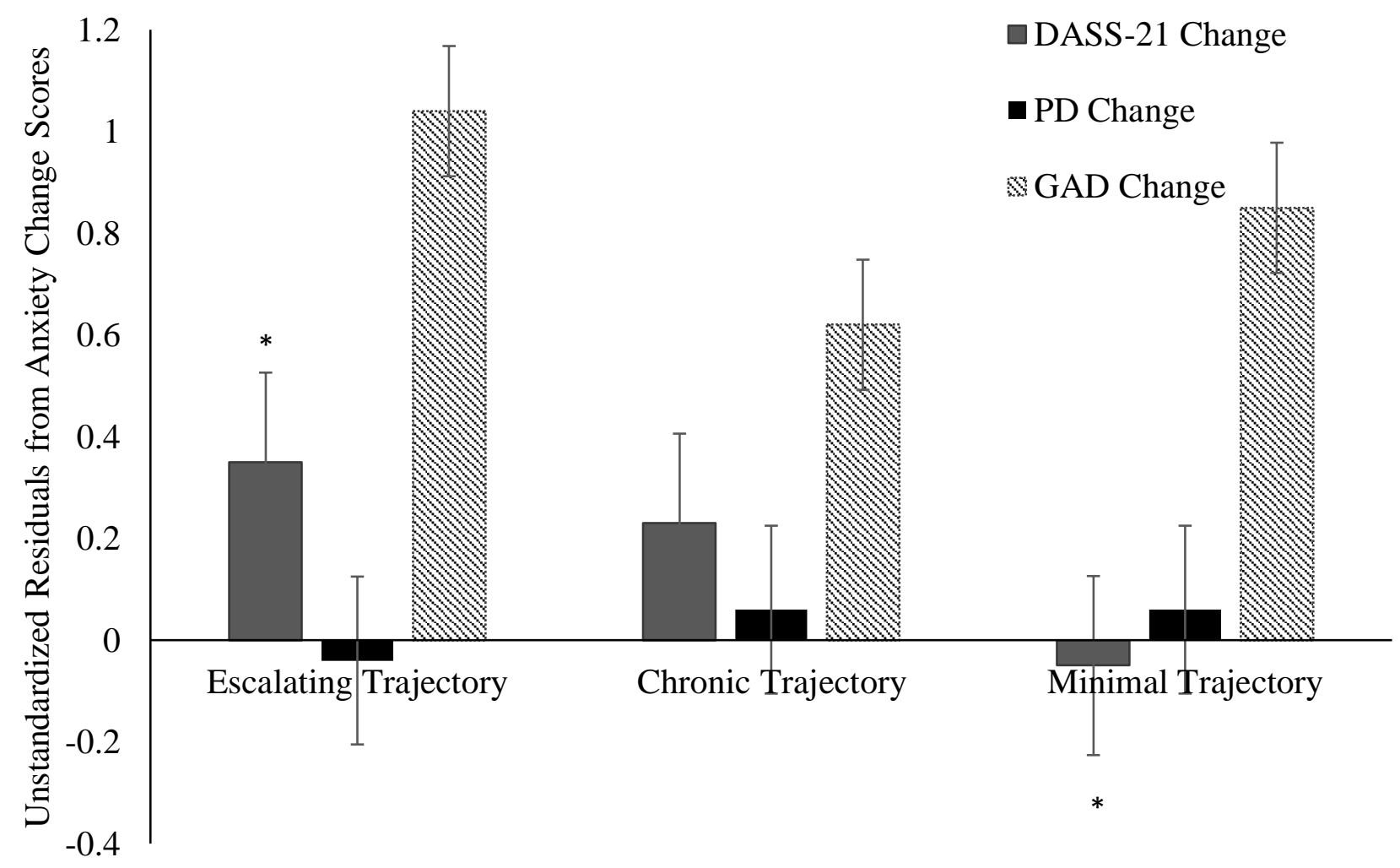

Cannabis Use Trajectories 
Figure 10: The Moderating Role of Anxiety on the Association Between Cannabis Use Trajectory and Decision-Making

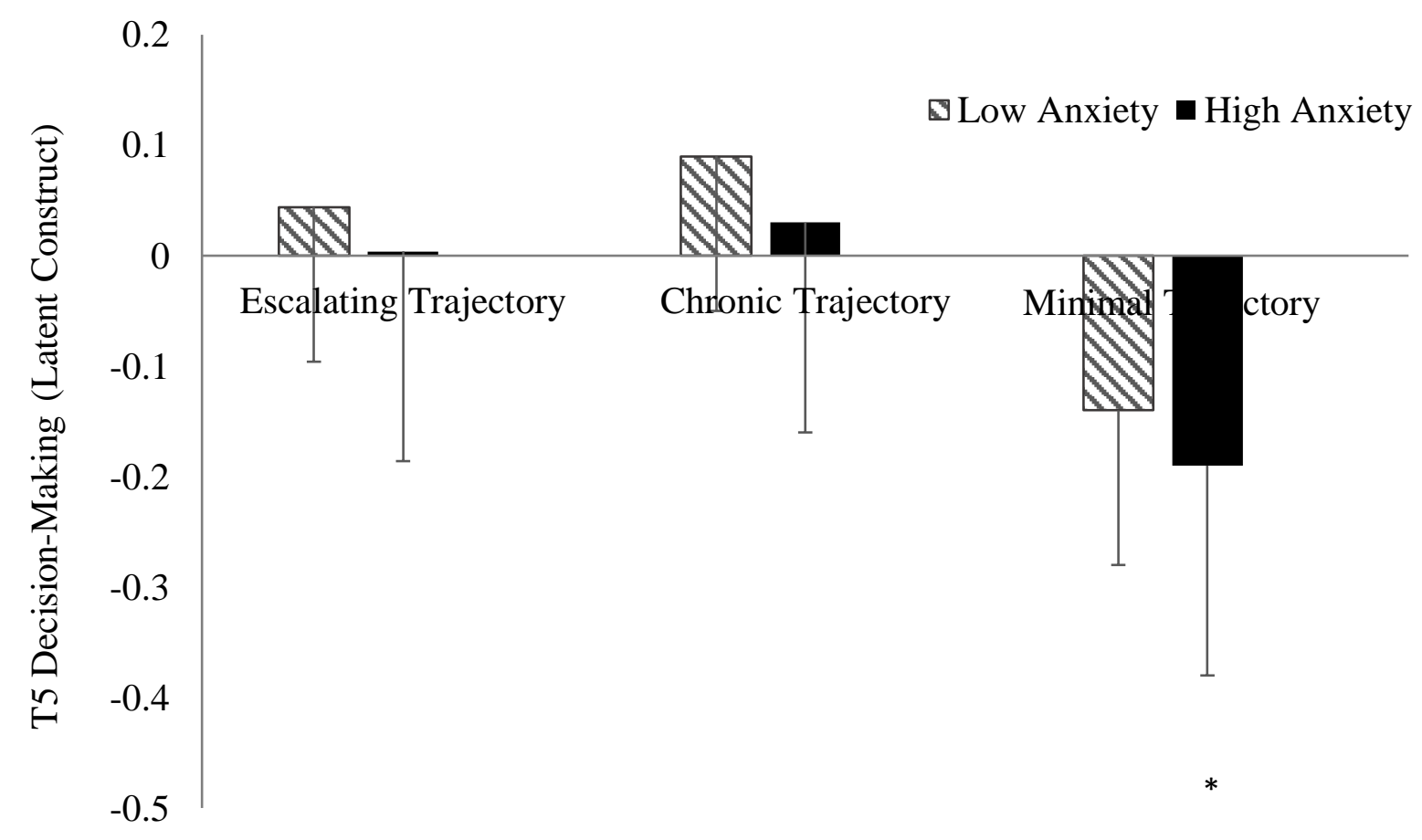

Cannabis Use Trajectories 


\title{
JACQUELINE CHRISTINE DUPERROUZEL
}

\author{
EDUCATION \\ 2019 - present $\quad$ Psychology Intern (Neuropsychology) \\ Nicklaus Children's Hospital, Miami, FL \\ 2014 - present $\quad$ Doctor of Philosophy in Psychology \\ (Majors: Clinical Science \& Cognitive Neuroscience) \\ Florida International University, Miami, FL, Department of Psychology \\ APA-accredited doctoral program; APCS Member program \\ 2014 - $2016 \quad$ Master of Science in Psychology \\ Florida International University, Miami, FL, Department of Psychology \\ APA-accredited doctoral program; APCS Member program \\ 2009 - 2013 Bachelor of Science in Psychology, minor in Biological Science \\ Florida State University, Tallahassee, FL, College of Arts \& Sciences \\ Summa cum laude, Honors College
}

\section{PEER-REVIEW PUBLICATIONS \& PRESENTATIONS}

Duperrouzel, J.C., Hawes, S. Pacheco- Colón, I., Granja, K., \& Gonzalez, R. (February, 2020). Associations among Adolescent Cannabis Use Trajectories, Anxiety, and Risky DecisionMaking. Oral paper presented at the 47th Annual Meeting of the International Neuropsychological Society, Denver, CO.

Duperrouzel, J.C., Granja, K., Pacheco-Colón, I., \& Gonzalez, R. (2019). Adverse Effects of Cannabis Use on Neurocognitive Functioning: A Systematic Review of Meta-Analytic Studies. Journal of Dual Diagnosis, 1-15.

Duperrouzel, J.C., Hawes, S., Lopez-Quintero, C., Pacheco- Colón, I., Coxe, S., Hayes, T., \& Gonzalez, R. (2019). Adolescent Cannabis Use and its Associations with Decision-Making and Episodic Memory: Preliminary Results from a Longitudinal Study. Neuropsychology.

Duperrouzel, J.C., Pacheco- Colón, I., Hawes, S., \& Gonzalez, R. (February, 2019). No evidence for additive adverse effects of alcohol and cannabis use on memory performance during adolescence. Poster presented at the 46th Annual Meeting of the International Neuropsychological Society, New York, NY. 
Pacheco-Colón, I., Hawes, S. W., Duperrouzel, J. C., Lopez-Quintero, C., \& Gonzalez, R. (2019). Decision-Making as a Latent Construct and its Measurement Invariance in a Large Sample of Adolescent Cannabis Users. Journal of the International Neuropsychological Society, $1-7$.

Duperrouzel, J.C., Lopez-Quintero, C., Granja, K., Pacheco- Colón, I., Hawes, S., Gonzalez, R. (June, 2018). Does anxiety moderate the effects of cannabis use on decision-making? Poster presented at the 16th Annual Conference of the American Academy of Clinical Neuropsychology, San Diego, CA.

Hawes, S.W., Trucco, E.M., Duperrouzel, J. C., Coxe., S., \& Gonzalez, R. (2018).

Developmental Pathways of Adolescent Cannabis Use: Risk Factors, Outcomes and Sex-Specific Differences. Substance Use and Misuse, 1-11.

Ross, J.M., Granja, K., Duperrouzel, J.C., Pacheco-Colón, I., Lopez-Quintero, C., Hawes, S., \& Gonzalez, R. (2018). Risky sexual behavior among adolescents: The role of decisionmaking, cannabis use and externalizing disorder symptoms. Journal of Clinical and Experimental Neuropsychology, 1-12.

Lopez-Quintero, C., Granja, K., Hawes, S., Duperrouzel, J.C., Pacheco-Colón, I., Gonzalez, R. (2018). Transition to drug co-use among adolescent cannabis users: The role of decision-making and mental health. Addictive Behaviors, 85, 43-50.

Duperrouzel, J.C., Hawes, S., Lopez-Quintero, C., Pacheco- Colón, I., Comer, J., \& Gonzalez, R. (2018). The association between adolescent cannabis use and anxiety: A parallel process analysis. Addictive behaviors, 78, 107-113.

Pacheco- Colón, I., Coxe, S., Musser, E. D., Duperrouzel, J. C., Ross, J. M., \& Gonzalez, R. (2018). Is Cannabis Use Associated with Various Indices of Motivation among Adolescents?. Substance use \& misuse, 53(7), 1158-1169.

Duperrouzel, J.C., Pacheco- Colón, I., Lopez-Quintero, C., Hawes, S., Gonzalez, R. (June, 2017). Material-specific sex differences in relationships between cannabis use and episodic memory among adolescents. Poster presented at the 79th Annual Meeting of the College on Problems of Drug Dependence, Montreal, QB, Canada.

Gonzalez, R., Pacheco- Colón, I, Duperrouzel, J.C., \& Hawes, S. (2017). Does Cannabis Use Cause Declines in Neuropsychological Functioning? A Review of Longitudinal Studies. Journal of the International Neuropsychological Society, 23 (9-10), 893-902. 